\title{
VACÍOS EN LA LITERATURA SOBRE FILOSOFÍA DE LA EDUCACIÓN AMBIENTAL: UNA REVISIÓN DE LAS CORRIENTES DE LA FILOSOFÍA AMBIENTAL
}

\author{
SERGIO ÁNGEL BAQUERO ${ }^{1}$
}

Recibido el 1 de agosto de 2012 y aprobado el 19 de diciembre de 2012

\section{RESUMEN}

El presente artículo es un producto preliminar del proyecto de investigación "Una propuesta de lineamientos para la formación ambiental en la educación superior colombiana", adelantado con la Universidad Antonio Nariño durante los años 2011 y 2012. Son dos los objetivos que se persiguen: por un lado, demostrar el vacío de la literatura sobre filosofía de la educación ambiental; y por otro lado, mostrar que a pesar de que las reflexiones epistemológicas sobre filosofía ambiental son pobres, la literatura sobre corrientes de la filosofía ambiental es prolífica. De manera que el orden de la exposición recurre, primero, a una revisión de la literatura sobre filosofía de la educación ambiental; segundo, a una revisión de la literatura sobre filosofía ambiental; y tercero, a un análisis de las principales corrientes, a saber, la ecología profunda, la biología de la conservación, el biorregionalismo y el ecofeminismo.

\section{PALABRAS CLAVE}

Ecología profunda, biología de la conservación, biorregionalismo, ecofeminismo.

\section{GAPS IN ENVIRONMENTAL EDUCATION PHILOSOPHY LITERATURE: A REVIEW OF TRENDS IN ENVIRONMENTAL PHILOSOPHY}

\section{ABSTRACT}

This article is a preliminary product of the research project "A proposal of guidelines for environmental training in Colombian Higher Education" carried out with Universidad Antonio Nariño during the years 2011and 2012. There are two objectives to be achieved: to demonstrate the void of literature on environmental education philosophy and to show that, despite the epistemological reflections on environmental philosophy are poor, the literature on trends in environmental philosophy is prolific. Thus, the order of the presentation is, first, a literature review on environmental education philosophy;, secondly, a literature review on environmental philosophy; and in third place, an analysis of the main trends, namely, deep ecology, conservation biology, bioregionalism and ecofeminism. 


\section{KEY WORDS}

Deep ecology, conservation biology, bioregionalism, ecofeminism.

\section{INTRODUCCIÓN}

El presente artículo es un producto preliminar del proyecto de investigación "Una propuesta de lineamientos para la formación ambiental en la educación superior colombiana", adelantado con la Universidad Antonio Nariño durante los años 2011 y 2012. El objetivo que se persigue con el proyecto, grosso modo, es definir unas bases filosóficas, epistemológicas, pedagógicas y didácticas del deber ser de la formación ambiental, con el fin de diseñar y proponer lineamientos generales que sirvan de plataforma a la intervención en las estructuras curriculares de las universidades. Razón por la cual, teniendo en cuenta que el presente artículo se enfoca específicamente en la filosofía ambiental, es preciso aducir que el interés por esta área está intrínsecamente relacionado con la necesidad de identificar las concepciones ambientales de los diferentes actores. Concepciones, que a continuación serán presentadas atendiendo a una revisión bibliográfica y bibliométrica, que dé cuenta de las tendencias en la literatura.

Dicho esto, el presente artículo tiene por objeto indagar por la producción académica de las últimas dos décadas en materia de filosofía ambiental. Razón por la cual, la hipótesis de la que se parte es que existe un notable vacío en la literatura sobre el tema que lleva a pensar que, o bien, la producción se ha orientado a las corrientes filosóficas particulares, o no hay un interés manifiesto por la filosofía dentro de los estudios ambientales. Ciertamente, esta segunda alternativa queda rechazada de plano, de manera que el presente artículo se ocupa de mostrar que son pocos los trabajos que se concentran explícitamente en reflexiones epistemológicas sobre la filosofía ambiental, mientras que es prolífica la literatura que toma como referencia alguna de las corrientes de la filosofía ambiental. En este sentido, es interés de las líneas que siguen identificar las tendencias de la literatura en materia de filosofía ambiental, buscando con ello evidenciar las concepciones ambientales preponderantes dentro de los estudios ambientales.

En este sentido, buscando demostrar la hipótesis antes planteada, el orden a seguir en el artículo será, primero, la descripción de las corrientes de la filosofía de la educación ambiental con base en los pocos trabajos que se han producido sobre el tema; segundo, la elaboración de un estado del arte de la filosofía ambiental con base en los artículos publicados en revistas Scopus; y tercero, la realización de un estudio bibliométrico de las tendencias principales de la filosofía ambiental. 


\section{Trazos de una filosofía de la educación ambiental}

Uno de los estudios recientes de mayor importancia en el tema de la filosofía de la Educación Ambiental fue publicado por Pierre Walter (2009) de la University of British Columbia bajo el título de "Philosophies of Adult Environmental Education". En este trabajo el autor presenta una tipología de las tradiciones filosóficas de la educación ambiental en adultos con base en la clasificación que Elias y Merriam (1995) presentan en su libro Philosophical foundations of adult education. De manera que, siguiendo a Walter, son tres las razones por las que se eligieron estas tipologías, a saber:

(a) to allow a wider consideration of approaches to theorizing adult environmental education than currently exists; (b) to allow adult educators within the vast, yet largely undocumented, field of "green" educational practice to begin to locate themselves within particular philosophies of environmental education; and (c) to stimulate questioning and debate about philosophies of environmental education for adults, about practice in the field, and the connections between the two. (Walter, 2009, p. 4)

De este modo, siguiendo a Walter se puede decir que hay cinco tipos de filosofía de la educación ambiental para adultos: la liberal, la progresista, la conductista, la humanista y la radical. Algo que sin duda resulta inusitado debido a que el trabajo de Elias y Merriam solo se había aplicado en la educación para adultos en los campos de la extensión educativa, la enseñanza de idiomas, la educación empresarial y la capacitación en seguridad radioactiva (Walter, 2009). De este modo, el trabajo de Walter en la reflexión que aquí se adelanta cobra particular importancia en la medida que, aun cuando no interesa la educación para adultos, sí interesan las tipologías de educación ambiental. En consecuencia, teniendo en cuenta este criterio, a continuación se presentarán en conformidad con el trabajo de Walter las cinco filosofías de la educación ambiental, junto con la propuesta educativa, las estrategias de enseñanza y la evaluación del aprendizaje de cada una. Es de anotar que la información contenida en la siguiente Tabla fue extraída del artículo en mención, solo que al dejar de lado lo relacionado con la educación para adultos se suprimieron algunas columnas de la versión original elaborada por Walter. 
Tabla 1. Filosofías de la educación ambiental (Pierre Walter)

\begin{tabular}{|c|c|c|c|}
\hline Filosofias & Propuesta educativa & $\begin{array}{l}\text { Estrategias de } \\
\text { enseñanza y rol del } \\
\text { instructor }\end{array}$ & $\begin{array}{l}\text { Evaluación del } \\
\text { aprendizaje }\end{array}$ \\
\hline Liberal & $\begin{array}{l}\text { - Expansión del } \\
\text { conocimientoy la } \\
\text { formación intelectual. } \\
\text { - Desarrollo del } \\
\text { sentido espiritual, } \\
\text { moral y estético de los } \\
\text { ciudad anos. }\end{array}$ & $\begin{array}{l}\text { - El profesor es la } \\
\text { fuente de } \\
\text { conocimientoy } \\
\text { autoridad: el oráculo. } \\
\text { - Guías de aprendizaje } \\
\text { a través de } \\
\text { contenidos. } \\
\text { - Pedago gía centrada } \\
\text { en el libro asignado. } \\
\text { - Diálogo socrático } \\
\text { sobre las lecturas. } \\
\text { - Centrado en el } \\
\text { currículo de las artes } \\
\text { liberales. }\end{array}$ & $\begin{array}{l}\text {-Exámenes dela } \\
\text { materia. } \\
\text {-Ensayos. } \\
\text {-Exposición. }\end{array}$ \\
\hline Progresista & $\begin{array}{l}\text { - Educación para la } \\
\text { democracia y la } \\
\text { reforma social. } \\
\text { - Liberación de } \\
\text { talentos, experiencias } \\
\text { y conocimientos. } \\
\text { - Mejoramiento de la } \\
\text { condición humana. }\end{array}$ & $\begin{array}{l}\text { - Profesor como guía y } \\
\text { facilitador. } \\
\text { - Centrado en el } \\
\text { estudiante. } \\
\text { - Aprendizaje } \\
\text { experimental. } \\
\text { - Aprendizaje basado } \\
\text { en problemas de } \\
\text { aprendizaje. } \\
\text { - Centrado en el } \\
\text { método científico. }\end{array}$ & - Demostración. \\
\hline
\end{tabular}




\begin{tabular}{|c|c|c|c|}
\hline Conductista & $\begin{array}{l}\text { - Asegurar la } \\
\text { sobrevivenciay } \\
\text { minimizar el } \\
\text { sufrimiento de las } \\
\text { especies humanas. } \\
\text { - Controlar el } \\
\text { ambiente de } \\
\text { aprendizaje para } \\
\text { disminuir la tendencia } \\
\text { hacia la auto- } \\
\text { gratificación, agresión } \\
\text { y destrucción. }\end{array}$ & $\begin{array}{l}\text {-Administrador de la } \\
\text { contingencia. } \\
\text { - Ingeniero de } \\
\text { comportamiento. } \\
\text { - Ambiente deseado } \\
\text { de comportamiento. } \\
\text { - Recompensasy } \\
\text { castigos por cumplir } \\
\text { los objetivos de } \\
\text { comportamiento. }\end{array}$ & $\begin{array}{l}\text { - Observar los } \\
\text { cambios de } \\
\text { comportamiento. } \\
\text { - Resultad os medibles } \\
\text { basados en la } \\
\text { evidencia. } \\
\text { - Referencia a } \\
\text { criterios. }\end{array}$ \\
\hline Humanista & $\begin{array}{l}\text {-Auto-actualización. } \\
\text { - Trascendencia. } \\
\text { - Felicidad humana. } \\
\text { - Desarrollo social, } \\
\text { emocional, espiritual e } \\
\text { intelectual. }\end{array}$ & $\begin{array}{l}\text { - Facilitador: establece } \\
\text { clima de aceptación; } \\
\text { promueve el } \\
\text { crecimiento personal y } \\
\text { la conciencia de sí } \\
\text { mismo; usa las } \\
\text { experienciasy } \\
\text { potencialidades de los } \\
\text { alumnos. } \\
\text { - El aprendizaje es } \\
\text { holístico, subjetivoy } \\
\text { centrado en } \\
\text { problemas y tiene } \\
\text { lugar a través de la } \\
\text { cooperacióny el } \\
\text { trabajo en equipo. } \\
\text { - Alumnos tienen la } \\
\text { responsabilidad de su } \\
\text { aprendizaje. } \\
\text { - Centrado enun } \\
\text { currículo humanista. }\end{array}$ & $\begin{array}{l}\text { - Autoevaluación. } \\
\text { - Lograr resolver } \\
\text { problemas. } \\
\text { - Contratos } \\
\text { individuales de } \\
\text { aprendizaje. }\end{array}$ \\
\hline
\end{tabular}




\begin{tabular}{|c|c|c|c|}
\hline Radical & $\begin{array}{l}\text { - Liberación de la } \\
\text { opresión social, } \\
\text { económica y política. } \\
\text { - Humanización de los } \\
\text { alumnosy la } \\
\text { sociedad. } \\
\text { - Transformación } \\
\text { social. }\end{array}$ & $\begin{array}{l}\text { - Facilitador, } \\
\text { coinvestigador, } \\
\text { organizador, activista. } \\
\text { - Pedagogía del } \\
\text { oprimido. } \\
\text { - Diálogo, reflexióny } \\
\text { acción ("praxis } \\
\text { dialógica"). } \\
\text { - Planteamiento de } \\
\text { problemas de } \\
\text { educación. } \\
\text { - Centrado en "temas } \\
\text { generadores" y } \\
\text { "lecturas del mundo". }\end{array}$ & $\begin{array}{l}\text {-Aumento de la } \\
\text { conciencia crítica. } \\
\text { - Acciones políticas. } \\
\text {-Cambios sociales } \\
\text { visibles. }\end{array}$ \\
\hline
\end{tabular}

Fuente: Basado en Walter (2009).

Tomando como referencia la Tabla 1 se pueden establecer tres campos de diferenciación entre las filosofías de la educación para adultos, a saber, la propuesta educativa, las estrategias de enseñanza y la evaluación del aprendizaje. En este sentido, antes de establecer inferencias acerca de la filosofía de la educación ambiental, propiamente dicha, se presentará una diferenciación en estos tres campos.

Dicho lo anterior, tomando como referencia la propuesta educativa es posible afirmar que cada una de las filosofías de la educación hacen énfasis en aquellos elementos que parecen sobresalientes desde una óptica particular. Es el caso, por ejemplo, de la educación liberal en donde la propuesta educativa propende por una ampliación de los conocimientos acompañada por una formación consistente con la ética ciudadana. Aspectos que contrastan sin duda con el énfasis que la filosofía radical pone sobre la liberación de la opresión social con el propósito de propiciar una transformación social y una humanización de los estudiantes. En otras palabras, mientras que el modelo liberal propende por mantener inquebrantables las estructuras sociales vigentes, el modelo radical propende por transformarlas, así sea a través de la conciencia de los estudiantes (ver Tabla 1).

Ahora bien, en relación con las estrategias de enseñanza es preciso aducir que el modo como se pretende transmitir el conocimiento varía de acuerdo con los propósitos que se persiguen. Es así que mientras la filosofía liberal puede transitar entre estrategias como el diálogo socrático, también puede apelar al libro de texto o quizás a la consideración del docente 
como oráculo. Ciertamente estas estrategias se podrían recusar como antagónicas, pero teniendo en cuenta las consideraciones de Walter (2009) es posible afirmar que en función de ampliar el conocimiento cualquiera de estas estrategias resulta funcional. Algo similar a lo que sucede con las demás filosofías, pues en el caso de la progresista, por ejemplo, al buscar una educación para la reforma social en la que se liberen los talentos, experiencias y conocimientos, sin duda la estrategia no puede ser otra que un docente facilitador y una enseñanza centrada en el estudiante (ver Tabla 1).

En este orden de ideas, siguiendo con lo anterior, el último criterio para tomar en consideración es la evaluación del aprendizaje. Al respecto se puede señalar que las evidencias del aprendizaje varían de acuerdo con los propósitos y las estrategias, así que mientras la filosofía liberal se avoca por exámenes, ensayos o exposiciones en los que el estudiante muestra suficiencia en la materia; la filosofía humanista, en donde se propende por la autorrealización, se concentra en una evaluación más personal y de autorreconocimiento, es decir, una autoevaluación. Aspecto que contrasta sin duda con la evaluación de la filosofía radical centrada en el aumento de la conciencia crítica y los cambios sociales visibles. En suma, mientras que la primera de las filosofías, antes mencionadas, pretende un mayor conocimiento sobre el tema, la segunda se avoca por una mayor realización del estudiante y la tercera por una mayor conciencia crítica (ver Tabla 1).

Luego de presentar una descripción general de la Tabla 1, es preciso atender ahora a las diferentes tipologías filosóficas de la educación ambiental. Al respecto es posible señalar que hay un vacío en relación con el propósito de este artículo, pues en la Tabla se deja de lado la comprensión sobre el ambiente. Así que, siguiendo a Walter, se puede sostener que en relación con la concepción liberal es previsible pensar que autores como Alice Hamilton o Rachel Carson abogan por una visión en la que el conocimiento -en este caso la educación- tiene el poder de transformar la comprensión y apreciación que, de la naturaleza, tienen los adultos. Lo cual lleva a suponer que esta perspectiva parte de la máxima socrática, según la cual, un mayor conocimiento del ambiente se traduce en mayor preservación y mayor protección. Es el caso, por ejemplo, del exvicepresidente de Estados Unidos Albert Gore que a través de su documental "Una verdad incómoda" (2006) buscaba aumentar la conciencia pública sobre el calentamiento global (Walter, 2009).

En cuanto a la concepción progresista el autor anota que la influencia sobre la educación ha sido notoria desde comienzos del siglo XX a través de las enseñanzas de Gifford Pinchot, Aldo Leopold y George Perkins. Se dice que la educación ambiental con matices progresistas ha tenido un fuerte influjo de los movimientos ambientalistas y por ende las prácticas educativas han estado orientadas hacia la educación al aire libre. Es de anotar que en la praxis los movimientos ambientalistas de talante progresista han dado una lucha en contra de la explotación indiscriminada de los recursos 
naturales, promoviendo la planificación, racionalización y eficiencia en el uso de bosques, tierras y aguas. Mientras que la concepción conductista en contravía con la concepción progresista supone que no es suficiente con la experiencia de la naturaleza, y en su condición de teoría psicológica de la educación pone el énfasis en el poder del ambiente construido por el ser humano como determinante del comportamiento social, individual y colectivo. En otras palabras, la educación debe ser promovida a través de estímulos de tal manera que se deben propiciar ciertos comportamientos ambientales. Tal y como Skinner lo sostiene, al afirmar que los comportamientos deseados se promueven a través de recompensas y los comportamientos no deseados se extinguen a través de castigos (Walter, 2009).

La concepción humanista, en contraste con la visión conductista, enfatiza el aprendizaje autodidacta sobre la base de las necesidades, los intereses y los deseos de los alumnos. De manera que, en conformidad con los planteamientos de los psicólogos Carl Rogers y Abraham Maslow, la responsabilidad del aprendizaje recae plenamente en el estudiante. De tal forma que, como lo sostiene Walter, en relación con la conexión espiritual entre el hombre y la naturaleza: "the humanist philosophical tradition is based on a belief in the wisdom of nature as integral to human spirituality, identity, and, indeed, existence" (Walter, 2009, p. 17). Algo que sin duda, se separa de la concepción radical en la medida que esta vertiente, a diferencia del humanismo no se centra en el desarrollo del individuo, sino que promueve la concienciación y la acción colectiva en orden a lograr el cambio social. Es por ello que la concepción radical de la educación ambiental con base en los presupuestos de La pedagogía del oprimido de Paulo Freire (1970), supone un activismo dentro de los movimientos de liberación en orden a conseguir que sus miembros aprendan a liberarse del yugo ideológico y material de las estructuras sistémicas (Walter, 2009).

Por todo lo anterior, es posible reconocer que el trabajo de Walter representa un punto de partida importante en la reflexión acerca de la filosofía de la educación ambiental. Aunque su trabajo es propedéutico, sobre todo en relación con las inferencias sobre la educación ambiental, permite pensar las implicaciones que cada una de estas tradiciones filosóficas tiene sobre la concepción del ambiente y la educación. En palabras del mismo autor:

Each philosophical tradition of environmental adult education is in itself a fertile site for further research, whether this is on beliefs about the nature of adult learning, the role of the instructor, the teaching-learning transaction, or the impact of learning within the environmental movement and larger society beyond. Case studies would be very valuable, for instance, on non formal education in zoos, nature centers, aquariums, and so on in the liberal tradition; on outdoor education or adult learning and 
ecotourism in the progressive tradition; on corporate "greening" education and public environmental education campaigns in the behaviorist tradition; on the learning that takes place within humanistic environmental education in deep ecology or spiritual ecology; and on further instances of learning and education within environmental protest in the radical tradition. (Walter, 2009, p. 21)

Pero, aun cuando el trabajo de Walter es de destacada trascendencia dentro del campo de la filosofía de la educación ambiental, es preciso anotar que no es el único trabajo sobre el tema. Park (1984), por ejemplo, en su artículo "Towards a philosophy of environmental education" explora la necesidad de otorgar un fundamento filosófico a la educación ambiental, con el fin de buscar que la formación en este ámbito propenda por cambiar las actitudes y los comportamientos de la comunidad hacia el medio ambiente. Al respecto el autor arguye que son dos los ingredientes básicos de una filosofía sensata y apropiada que permiten este propósito: de un lado, la necesidad de evitar el adoctrinamiento y de concentrarse en el desarrollo de actitudes y la adquisición de conocimientos; y de otro lado, la necesidad de ofrecer una visión positiva, optimista y realista de los temas ambientales. Es así como el artículo de Park en orden a transformar las actitudes de la ciudadanía cimenta las bases de la filosofía ambiental en el marco de la ética (Park, 1984).

De igual modo, Manuela Gómez e Irene de Puig (2003) en su artículo "Ecodiálogo, environmental education and philosophical dialogue" buscan fortalecer la educación ambiental en la escuela primaria y secundaria por medio de la reflexión filosófica fundada en bases interdisciplinarias e interculturales. A juicio de las autoras la filosofía permite aclarar las preguntas que no tienen una respuesta definitiva y sobre las cuales la filosofía ha hecho aportes importantes, como por ejemplo los conceptos de naturaleza y cultura, permanencia y cambio, realidad y apariencia, libertad y responsabilidad, entre otros. Pero, esta no es la única tarea de la filosofía dentro de la educación ambiental, pues esta también permite cuestionar las ideas que hasta ahora se han dado por ciertas y que no han sido pensadas suficientemente o simplemente no han sido pensadas (Gómez \& De Puig, 2003).

Pero Gómez y De Puig no se quedan en el cuestionamiento a los conceptos e ideas de la práctica ambiental, sino que van más allá y centran su atención en el componente ético, llegando a afirmar que las relaciones que los seres humanos sostienen con el ambiente son medibles en las ideas y por ende en las concepciones que se tienen sobre el mundo. Así que trabajar con el medio ambiente significa trabajar con las ideas. De tal manera que, para transformar las actitudes frente al ambiente, es preciso que en la primaria y la secundaria se dé un diálogo entre la filosofía y la educación ambiental: 
To promote dialogue in education implies creating the conditions under which children participate in conversations which take the form of investigation disciplined by logic and metacognitive considerations. Some examples of the latter are: What is our relationship with nature? How do we know that a statement is true?; What makes one reason better than another? Is it possible for certain behavior to be at the same time legal and wrong? On the basis of what criteria is A better than B? etc. (Gómez \& De Puig, 2003, p. 37)

Lo cierto es que las autoras, siguiendo a Mathew Lipman, sostienen que la filosofía no presupone un discurso teorético, sino exacerbadamente práctico, en la medida que el modo de pensar propicia cambios en las actitudes y disposiciones frente al ambiente. Lo cual las lleva a sostener, finalmente, que la investigación filosófica requiere de un gran número de habilidades de pensamiento, tales como: estar abierto a la corrección de nuestros juicios, descubrir las implicaciones y consecuencias de nuestros puntos de vista, formular hipótesis y preguntas, y clarificar conceptos y formular juicios (Gómez \& De Puig, 2003).

En este orden de ideas, el último artículo encontrado en la búsqueda sobre filosofía de la educación ambiental fue escrito por Peter Corcoran y Eric Sievers (1994) y se titula "Reconceptualizing environmental education: five possibilities". En este artículo los autores describen algunas filosofías emergentes y su impacto sobre la educación ambiental, procurando con ello explorar el poder filosófico y metodológico de la educación ambiental en la solución de los problemas ambientales y educativos. Es así que los autores, establecen como punto de partida que la educación ambiental en Norteamérica no ha adoptado perspectivas filosóficas alternativas como la ecología profunda, la biología de la conservación, el biorregionalismo, el ecofeminismo y el análisis de la crítica social. Perspectivas que, a juicio de quienes escriben, resultan relevantes en un campo ávido de energía intelectual, emocional y espiritual, que está en la necesidad de llegar a nuevas audiencias, ampliar la definición de quienes educan en lo ambiental y de crear métodos más eficaces en su capacidad de producir cambios (Corcoran \& Sievers, 1994). En este sentido, la Tabla 2 permite identificar los aportes de las perspectivas filosóficas alternativas a la educación ambiental. 
Tabla 2. Filosofías de la educación ambiental (Peter Corcoran y Eric Sievers)

\begin{tabular}{|c|c|}
\hline Filosofias & Aportes a la educación ambiental \\
\hline Ecología profunda & $\begin{array}{l}\text { - Promueve un enfoque biocéntrico dela vida, que se esfuerza } \\
\text { porver a los humanos como parte integral de toda la creación, } \\
\text { pero no comouna creación ontológicamente más valiosa que } \\
\text { otras formas de vida. } \\
\text { - Ofrece un contrapunto al sentido moderno del lugar y la } \\
\text { epistemologia. Des de la perspectiva del estudiante, pone de } \\
\text { relieve la diferencia entre la observación dela naturaleza como } \\
\text { un espectador individual y la interacción como parte de una } \\
\text { comunidad dinamica de la naturaleza, } \\
\text { - Un número importante de los ecologistas profundos apelan a } \\
\text { la desobediencia civil para proteger la integridad biológica de la } \\
\text { tierra. Este tipo de acciones pueden desempeñar un papel } \\
\text { educativoprofundo. La acciónpersonal puede ampliar nuestra } \\
\text { comprensión de las cuestiones ambientales, incluso aclarar la } \\
\text { participación de uno en la sociedad como un vehículo para el } \\
\text { cambio. }\end{array}$ \\
\hline Biología de la conservación & $\begin{array}{l}\text { - Los biólogos de campo, en particular los que trabajan en } \\
\text { áreas de altas tasas de extinción exacerbada por la actividad } \\
\text { antropogénica, comenzaron a referirse así mismos en la } \\
\text { década de } 1970 \text { como biólogos de la conservación. Su deseo } \\
\text { de detener la actividad destructiva, preservar la diversidad } \\
\text { biológica y desarrollarun enfoque responsable y eficaz para la } \\
\text { conservación biótica dio lugar a este nuevo campo. } \\
\text { - Desde el punto devista de un educador ambiental, la biología } \\
\text { de la conservación es particularmente notable por su rápido } \\
\text { crecimiento, elocuencia, energía y consecuencias. El examen } \\
\text { de la pertinencia de las esferas políticas y sociales para el } \\
\text { desarrollo de estrategias de conservación exitosas, la discusión } \\
\text { de la relevancia de los sentimientos personales de gran parte } \\
\text { de los biólogos de la conservación y la discusión de la } \\
\text { importancia de la educación ambiental. } \\
\text { - La biología de la conservación se ofrece a los educadores } \\
\text { ambientales de manera palpabley sólida para la interrelación } \\
\text { con el mundo natural. Muchos biólogos de la conservación } \\
\text { están desarrollando una visión macroscópica científica de la } \\
\text { naturaleza. Ellos nosolo están estudiando como funcionan los } \\
\text { ecosistemasy cómo cada organismo o elemento encaja en el } \\
\text { mosaico de la vida, sino que también están reconociendo los } \\
\text { contextos socialesy económicos de esta información. Teniendo } \\
\text { en cuenta que una educaciónambiental para los biólogos de la } \\
\text { conservación debe seguir el ejemplo de Aldo Leopold, abogan } \\
\text { por la necesidad de una educación conservadora más y mejor. }\end{array}$ \\
\hline
\end{tabular}




\begin{tabular}{|c|c|}
\hline Biorregionalismo & $\begin{array}{l}\text { - El biorregionalismo llama a la sociedad humana a estar más } \\
\text { estrechamente relacionada con la naturaleza y para ser más } \\
\text { consciente de su localidad, de la vida, el lugar y la región. Es } \\
\text { una propuesta para conectar a las culturas humanas con los } \\
\text { sistemas naturales; para conocer el lugar en el que se habita } \\
\text { íntimamente; y para evitar distorsionar la Tierra a las demandas } \\
\text { de la humanidad. } \\
\text { - El biorregionalismo difunde un espíitu derespeto por el lugar } \\
\text { creando las condiciones en las que la educación puede mirar a } \\
\text { las culturas que vivieron en armonía con los lugares en el } \\
\text { pasado teniendo la esperanza de que en el futuro seva viviren } \\
\text { armonía con los sistemas naturales. Cualquier discusión sobre } \\
\text { el biorregionalismo debe acreditara los nativos como fuente de } \\
\text { inspiración para vivir plenamente dentro de los límites de un } \\
\text { lugar. } \\
\text { - Gran parte de la tradición de estudio de la naturaleza y la } \\
\text { educación ambiental confirma los lazos de la comunidad al } \\
\text { lugar. El estudio de la historia natural y la apreciación de la } \\
\text { naturaleza en el espiritu de Rachel Carson crea el sentido de } \\
\text { lugar (y el "sentido de la maravilla"), necesarias para la } \\
\text { conexión que pone de relieve una perspectiva biorregional. } \\
\text { - El biorregionalismo trae a la educación ambientalun profundo } \\
\text { respeto por el lugar, el respeto de la comunidad en ese lugar y } \\
\text { la comprensión de la interdependencia. Así como también } \\
\text { permite el rechazo de la educación antropocéntrica y atomista } \\
\text { que nos aleja de nuestro medio ambiente y de los demás. }\end{array}$ \\
\hline Ecofeminismo & $\begin{array}{l}\text { - Un tema central del ecofeminismo es la interconexiónentre el } \\
\text { abuso de la naturaleza y la opresión de las mujeres. El } \\
\text { ecofeminismo afirma los ritmos naturales del cuerpo femenino } \\
\text { como sincrónica a los ritmos de la tierra, celebrando la } \\
\text { fecundidad primordial del proceso reproductivo. Estas } \\
\text { ideologías comparten una fuerte crítica de la dominación } \\
\text { masculina, la violencia, y las jerarquías. } \\
\text { - Debido a que la educación en general, y la educación } \\
\text { ambiental en particular, está centrada en el hombre, es } \\
\text { importante la exploración del ecofeminismo en un salón de } \\
\text { clases. El estudio del ecofeminismo facilita el proceso de } \\
\text { enfrentar la dominación masculina que inhibe la experiencia } \\
\text { educativa de muchas mujeres. De tal manera que sea posible } \\
\text { hacer que los estudiantes, y las estudiantes, desafien el } \\
\text { pensamiento tradicional masculino propiciando un profundo } \\
\text { entendimiento de las implicaciones, sobre la naturaleza y la } \\
\text { sociedad, de nuestra culturacentrada en el hombre. Logrando } \\
\text { asídemostrarque atendiendo a los derechos de las mujeres se } \\
\text { puede responder a la salud de la naturaleza. }\end{array}$ \\
\hline
\end{tabular}




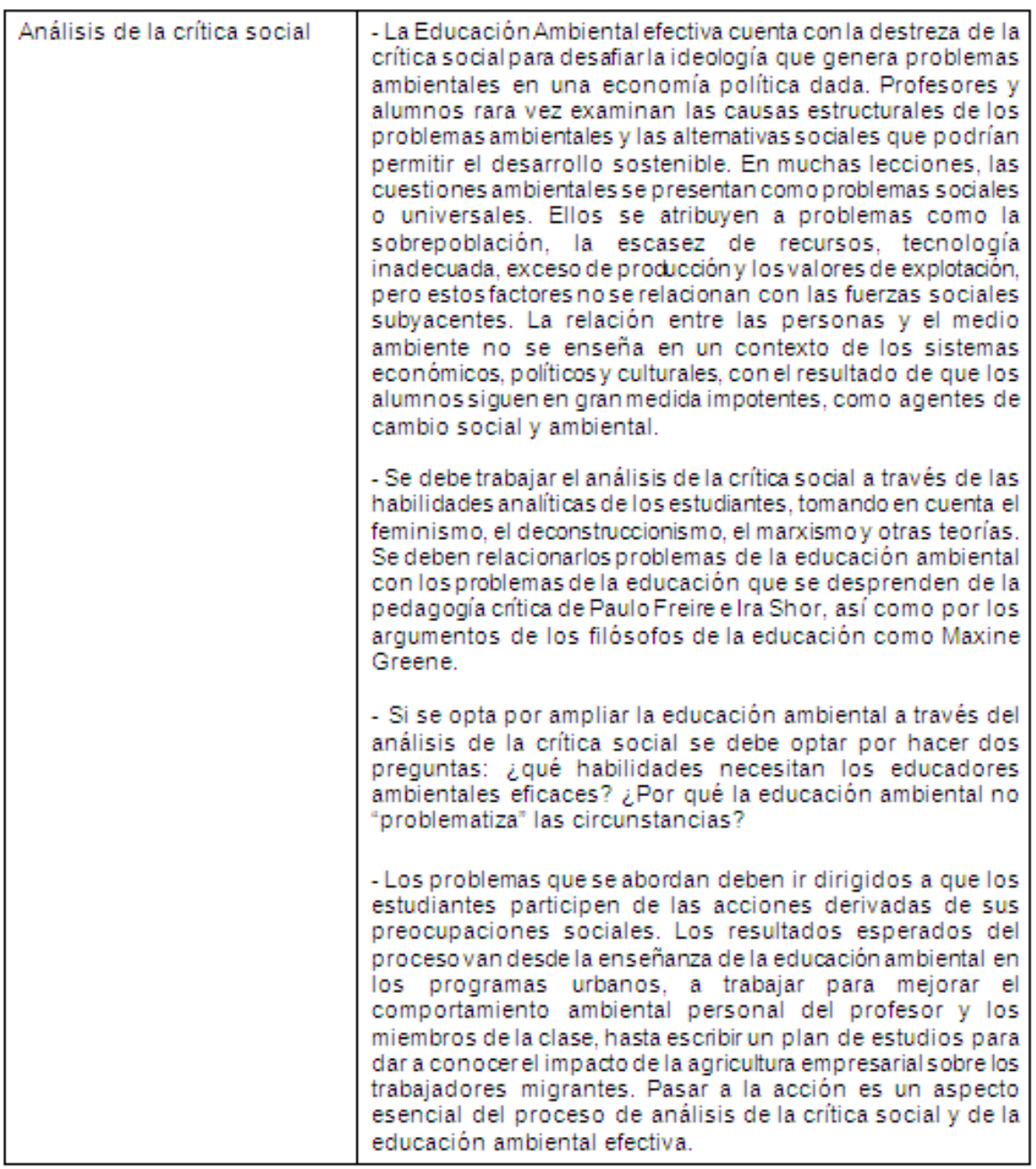

Fuente: Basado en Corcorany Sievers (1994).

El artículo de Corcoran y Sievers concluye diciendo que en el marco de los congresos y las reuniones internacionales de ambientalistas, se debe reconocer la importancia de las filosofías expuestas en orden a generar cambios sobre el modo como el ser humano se relaciona con el ambiente. Es así que los autores agregan que el medio ambiente tiene que ser reformulado, al ser ampliado por la ecología profunda, informado por las perspectivas de la biología de la conservación, puesto en contexto a través del biorregionalismo, enriquecido por el ecofeminismo y crítico a través del análisis de la crítica social (Corcoran \& Sievers, 1994).

En suma, por lo dicho anteriormente, se puede decir que la literatura sobre filosofía de la educación ambiental es incipiente y en realidad son pocos los aportes que hasta la fecha se han elaborado en la literatura en inglés. Sin embargo, la rezagada producción académica contrasta con la importancia del tema, pues como se vio la filosofía de la educación ambiental constituye un pilar fundamental de la formación ambiental y por ende de la efectividad de la misma. Ahora bien, a pesar de que los aportes de Walter (2009) y Corcoran \& Siever (1994) son de denotada importancia dentro de este campo, es de reconocer 
que resultan aislados dentro de la literatura sobre los estudios ambientales. Además, da la impresión de que sus trabajos se mantienen en los lugares comunes de la educación ambiental invitando a la consciencia ambiental y el respeto por el lugar.

La filosofía de la educación ambiental cobra importancia en la medida en que le otorga fundamentos a un modo de enseñar, pero también a un modo de entenderse en el ambiente, de manera que si sus fundamentos son pobres, o al menos, reiterativos y poco trascendentes, es factible que la formación resulte incipiente y se redunde en los problemas actuales de la educación. Es así que reconociendo la importancia de las concepciones ambientales en la transformación de la conducta ambiental, se hace necesario inquirir sobre la filosofía ambiental. Ciertamente, si el trabajo en filosofía de la educación ambiental no ha sido muy prolífico es factible que la filosofía ambiental pueda tener mejores resultados y desde allí repensar el proceso formativo en el marco de un currículo ambiental, razón por la cual el siguiente apartado se ocupará de este campo.

\section{Apuntes para un estado del arte de la filosofía ambiental}

Si se tiene en cuenta que la filosofía ambiental es el punto de referencia de cualquier concepción sobre el ambiente, resulta presumible pensar que la literatura sobre el tema es prolífica. Sin embargo, al realizar una búsqueda en Scopus bajo el criterio [ENVIRONMENTAL PHILOSOPHY], se logran identificar apenas 27 documentos publicados en las 5300 revistas de Ciencias Sociales y Humanidades. Ciertamente, estos resultados corresponden a una búsqueda por título, que excluye todos aquellos documentos en los que las palabras aparecen dentro del abstract y las key words, pues al realizar una búsqueda de esta manera el número se incrementa, llegando hasta 813 documentos. Sin embargo, el crecimiento exponencial en la búsqueda puede ser explicado si se toma en consideración que muchos de los artículos se refieren de manera tangencial a la filosofía ambiental y agrupan los dos nombres de manera aleatoria.

En este sentido, tomando como referencia los 27 documentos de la búsqueda por título, el presente apartado se propone organizar la literatura sobre filosofía ambiental en cinco ejes temáticos, a saber: (1) los textos que se centran en los enfoques filosóficos del ambiente; (2) los textos en los que se aborda el ambiente desde la perspectiva ética; (3) los textos en los que se relacionan filosofía y educación; (4) los textos en los que se habla de la filosofía ambiental dentro de contextos determinados; y (5) los textos en los que se alude a la filosofía y la gestión ambiental. Es preciso anotar, antes de continuar, que esta clasificación es producto de una revisión personal de los textos, en conformidad con los contenidos de cada uno de los artículos.

Así es que, tomando en consideración el orden antes planteado, se puede decir que el primer grupo de textos 
concentra su atención en los enfoques de la filosofía ambiental. Al respecto se encuentra el texto de De Laplante (2004) en el que el autor busca alejarse de las concepciones ortodoxas, aludiendo a una concepción ecológica del ambiente; el texto de Kennet (2007) que, en calidad de editorial del International Journal of Green Economics, busca explorar las bases filosóficas de la economía verde; el texto de Utsler (2009) en el que se atiende a un enfoque hermenéutico de la filosofía ambiental desde la perspectiva de Paul Ricoeur; el texto de Macauley (2005) que en una exploración del pensamiento de Empédocles, desde la perspectiva del "rizoma" de Deleuze y Guattari, se ocupa de demostrar que este filósofo presocrático proporciona un conocimiento temprano, pero importante, de cuestiones que siguen siendo ecológicamente relevantes hoy en día; el texto de Markus (2003a) en el que se aboga por la ecología profunda como una filosofía crítica del medio ambiente de la civilización tecnológica; y finalmente, los textos de Nash $(1999,2000)$ en los que se asocia el problema del medio ambiente con el problema de la mujer, desde la perspectiva del feminismo.

Ahora bien, a pesar de que el interés de este escrito se concentra en los enfoques de la filosofía ambiental, es menester anotar que la literatura sobre el tema aborda otros asuntos de importancia, como la ética ambiental. Al respecto se encuentran artículos en los que se atiende a la dicotomía entre ecocentrismo y antropocentrismo (Hotam, 2010); artículos en los que se hace referencia a la filosofía de la acción en orden a determinar un método para la toma de decisiones ambientales (Goralnik \& Nelson, 2011); artículos en los que se atiende a la ética en los aspectos sociales del desarrollo sostenible (Tyburski, 2008); y artículos en los que se deja en evidencia que la ética no está cumpliendo con su promesa de contribuir a la resolución de los problemas ambientales (Light, 2002).

Ciertamente estos son solo algunos de los trabajos que sobre el tema se han desarrollado, pues al restringir la búsqueda a la filosofía ambiental solo se extrajeron los artículos en los que se alude al tema en el sentido filosófico. Pero, a pesar de que esta selección de textos parece no decir mucho, es posible aducir dos argumentos en su defensa: primero, que el interés de este apartado no es indagar por la ética sino por la filosofía; y segundo, que los textos encontrados permiten inferir que la ética es uno de los ejes temáticos relevantes dentro del campo de la filosofía ambiental.

Dicho esto, es preciso atender ahora a los documentos en los que se aborda la filosofía ambiental en concordancia con la educación. Aunque el apartado anterior de este escrito se ocupaba explícitamente de la filosofía de la educación ambiental, resulta interesante observar que al buscar la literatura sobre filosofía ambiental, precisamente uno de los ejes temáticos es la educación. Al respecto se encuentran artículos en los que se apela a enfoques interdisciplinarios como medio para resolver los problemas ambientales (Thompson \& Whyte, 2011); artículos en los que se defiende la concepción ecológica del medio ambiente como un recurso 
para la enseñanza de los cursos de filosofía ambiental (De Laplante, 2004); artículos, en los que se indaga por las filosofías medioambientales de los estudiantes universitarios (Schultz, Simpson \& Elfessi, 2011); artículos en los que se habla de las tradiciones de la filosofía de la educación ambiental (Walter, 2009); artículos en los que se habla de recursos didácticos, como películas, para la enseñanza de la filosofía ambiental (Gold, Revill \& Haigh, 1996); y artículos en los que se observa el potencial de la filosofía ambiental como medio para revitalizar las humanidades dentro de la educación superior (Scott, 2004). Es menester agregar que los trabajos antes mencionados no son exclusivamente teóricos, pues mientras que el trabajo de Thompson y Whyte (2011) defiende el papel de la filosofía como medio para promover la educación ambiental interdisciplinar, el trabajo de Schultz, Simpson y Elfessi (2011) apela, más bien, a un estudio comparado de las filosofías ambientales de estudiantes matriculados en la carrera de Estudios Ambientales y en la carrera de Gestión de la Recreación de la Midwestern University.

En este orden de ideas, otro de los ejes temáticos en los que se puede agrupar la literatura encontrada corresponde a la filosofía ambiental en contextos determinados. En primer lugar, se encuentra el artículo de Pfister (2007) titulado "Environmental ethics and some probing questions for traditional Chinese philosophy"; en segundo lugar, el artículo de Plumwood (1999) titulado "The struggle for environmental philosophy in Australia"; y en tercer lugar, el artículo de James (1998) titulado "The construction of India in some recent environmental philosophy". Sin duda, aunque todos estos trabajos tienen en común que buscan hacer una revisión de la filosofía ambiental dentro de contextos determinados, es de destacar que India y China son dos culturales orientales y Australia aunque hace parte de la cultura occidental, guarda marcados rasgos ancestrales y corresponde a uno de los continentes más apartados del planeta. De manera que estos trabajos destacan, por un lado, la importancia metodológica de los estudios de caso; y por otro lado, la importancia de pensar el medio ambiente en relación con las culturas ancestrales y las culturas orientales.

El último eje temático de la filosofía ambiental es el que agrupa todos aquellos trabajos en los que, implícita o explícitamente, se aborda el asunto de la gestión. Están los trabajos en los que se atiende a la filosofía de la gestión ambiental en relación con el desarrollo económico de China (Li et al., 2010, 2011); aquellos en los que se habla de la "gestión adaptativa" y la filosofía de la política ambiental (Howarth, 2007); los que examinan las filosofías del control a la contaminación en Europa en relación con la legislación ambiental de los diferentes países (Lübbe-Wolff, 2001); los que se refieren a los "métodos multicriterio" para la evaluación del impacto ambiental (Parkin, 1992); los que atienden a los problemas de la tecnología agrícola en relación con el desarrollo sostenible (Anthony, 2010); y los que se refieren a las limitaciones de los índices ambientales (Inhaber, 1975). Ciertamente se podrá discutir que el criterio resulta abrupto para encerrar trabajos tan heterogéneos como el de Anthony (2010) y el de Lübbe-Wolff 
(2001) pero si se tiene en cuenta que la gestión ambiental se refiere a las acciones y estrategias para el cuidado del medio ambiente, se puede afirmar entonces que, aunque general, este criterio agrupa los diferentes trabajos, desde la legislación ambiental hasta la tecnología agrícola.

Teniendo en cuenta lo dicho hasta acá es posible inferir que la filosofía ambiental agrupa temas bastante heterogéneos. Se habla de gestión ambiental pero también se alude a la educación ambiental. Ciertamente esto significa que la filosofía tiene mucho que aportarle a los diferentes campos del medio ambiente, de tal manera que su ámbito no se restringe exclusivamente a lo teórico y reflexivo, sino también a lo práctico. Pero, aun cuando la literatura se pudo agrupar en cinco diferentes ejes temáticos, es preciso preguntarse: ¿Cuál de estos resulta más importante? Al respecto es posible apelar a dos criterios: de un lado, a lo que tiene que ver con el número de documentos por eje temático; y de otro lado, a lo que tiene que ver con el número de citaciones de cada artículo.

Tabla 3. Número de documentos por eje temático

\begin{tabular}{|l|c|}
\hline Eje temático & Número de documentos \\
\hline Enfoques filosoficos del ambiente & 7 documentos \\
\hline Etica ambiental & 4 documentos \\
\hline Educacion ambiental & 6 documentos \\
\hline Filosofia ambiental en contexto & 3 documentos \\
\hline Gestion ambiental & 7 documentos \\
\hline Total de documentos & 27 documentos \\
\hline
\end{tabular}

Fuente: Elaboración propia (2012).

De acuerdo con el número de documentos por eje temático es posible inferir que los enfoques filosóficos del ambiente agrupan el mayor número de artículos, mientras que los estudios de caso de la filosofía ambiental ostentan el menor número (ver Tabla 3). Definitivamente esto no quiere decir que un eje temático es más importante que el otro, aunque si se evidencia que la mayor preocupación en relación con la filosofía ambiental se concentra en las corrientes filosóficas. Con base en la muestra de los 27 artículos encontrados en Scopus se puede decir que el $26 \%$ de los documentos - una cuarta parte de la muestra aproximadamente- se refiere a las corrientes. Hecho que, más que representar la preponderancia de un eje temático sobre los demás, representa que las corrientes filosóficas son de notoria importancia dentro de la filosofía ambiental. Y es esto precisamente lo que se quiere evidenciar, pues el siguiente apartado se ocupará de presentar un estudio bibliométrico de las diferentes corrientes. 
Tabla 4. Numero de citaciones por artículo

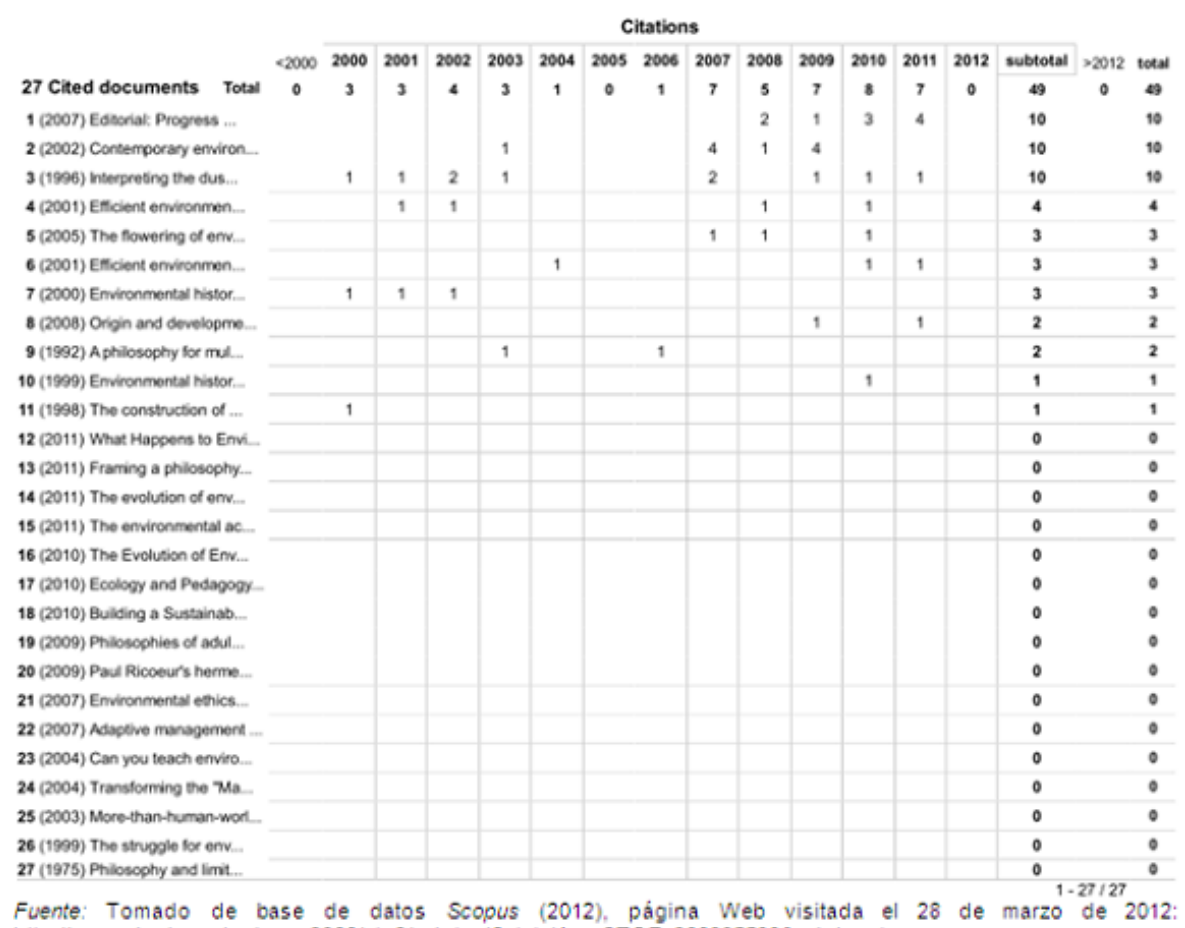

http://www.sinab.unal.edu.co:2066/cto $2 /$ print.url?stateKey $=C T O F \_288905593 \&$ rigin=cto

En cuanto a la citación de los documentos encontrados en Scopus vale la pena destacar tres artículos: en primer lugar, el trabajo publicado por Kennet (2007) que fue clasificado dentro de las corrientes filosóficas de la educación ambiental por referirse a las bases filosóficas de la economía verde; en segundo lugar, el trabajo publicado por Light (2002) que fue clasificado dentro de la ética ambiental debido a que mostraba las dificultades que la ética ha tenido al intentar responder a los problemas ambientales; y en tercer lugar, el trabajo de Gold, Revill y Haigh (1996) que fue clasificado dentro de la educación ambiental por referirse al uso didáctico de películas en la enseñanza de la filosofía ambiental. Comparativamente es de destacar el trabajo de Kennet en la medida que tiene el mismo número de citaciones de los otros dos (10 citas), pero fue escrito en el año 2007, mientras que los otros fueron escritos en el año 2002 y en el año 1996, respectivamente. Esto quiere decir que el trabajo de Kennet, en razón al número de citas y el año de publicación, es quizás el texto de mayor importancia dentro de la literatura examinada. Así que tomando como referencia el número de documentos de cada eje temático y el número de citaciones de cada artículo, se puede afirmar que las corrientes de la filosofía ambiental, no solo resultan relevantes hoy día, sino que además tienen una destacada importancia dentro de la filosofía ambiental.

\section{Corrientes de la filosofía ambiental}

De acuerdo con la hipótesis planteada en este artículo aunque existe un vacío notable en la literatura sobre filosofía ambiental, es plausible pensar que este hecho radica en la falta de 
reflexión epistemológica y no en el desinterés o el desprecio por la filosofía de los estudios ambientales. Esto es posible demostrarlo si al buscar de manera genérica los artículos sobre filosofía ambiental se hallan menos documentos que al buscar por cada una de las corrientes filosóficas. Así es que en orden a comparar los resultados del apartado anterior, se tomarán en consideración cuatro de las corrientes filosóficas expuestas por Peter Corcoran y Eric Sievers (1994), a saber: la ecología profunda, la biología de la conservación, el biorregionalismo y el ecofeminismo. Es de anotar que fueron dejados de lado los criterios de Walter (2009) por ser demasiado generales y no referirse específicamente al campo ambiental (filosofía liberal, progresista, conductista, humanista y radical). Así como también fueron dejados de lado enfoques como la hermenéutica ambiental (Utsler, 2009), la economía verde (Kennet, 2007) y el posestructuralismo ambiental (Macauley, 2005), al presentar trabajos aislados y no atender a reflexiones filosóficas explícitas.

En este sentido, a continuación se llevará a cabo un estudio bibliométrico por cada una de las corrientes ambientales. Atendiendo, en primer lugar, al número de publicaciones por año; en segundo lugar, al número de publicaciones por autor; y en tercer lugar, al número de publicaciones por país. Todo esto con el fin de analizar comparativamente el desarrollo de cada una de las corrientes ambientales.

\section{Ecología profunda}

Teniendo en cuenta que en el primer apartado de este escrito se expuso la ecología profunda en conformidad con el trabajo de Corcoran y Sievers (1994), a continuación, más que desarrollar el marco conceptual de esta corriente de la filosofía ambiental, se buscará indagar por el desarrollo que esta ha tenido durante los últimos años. Para comenzar, es preciso anotar que la búsqueda se concentró en los 5300 títulos de las revistas de Ciencias Sociales y Humanidades bajo el criterio de [Deep_ecology]. Pero a diferencia del apartado anterior, la búsqueda presenta dos modificaciones: por una parte, en lugar de atender a todos los tipos de documentos -incluidos artículos, revisiones, conferencias, editoriales, etc.-, se tomaron en consideración solamente los artículos; y por otra parte, en lugar de revisar si el criterio de búsqueda aparece en el título, se buscó que este apareciera también en el resumen y en las palabras clave. Estos dos cambios se deben, fundamentalmente, a que el estudio bibliométrico para ser pertinente reclama una muestra más grande de aquella que se puede conseguir a través de la búsqueda por el título. Además que los documentos que parecían no ser pertinentes en el apartado anterior, cobran relevancia en este dado que la búsqueda es mucho más amplia y puede incluir artículos en los que no se hace una reflexión explícita sobre la deep ecology.

En este sentido, con base en los criterios antes señalados, en una búsqueda realizada el día 28 de marzo de 2012 se encontró que aparecen registrados 58 artículos en un rango que 
se extiende desde el año 1985 hasta el año 2012. Esto quiere decir que el primer artículo en el que se habla de ecología profunda (al menos en los registros de Scopus) fue publicado en 1985, aunque el auge de esta corriente no se dará sino hasta mediados de los años noventa, y el punto más alto de la producción en el año 2002 con la publicación de 8 artículos. Es de destacar, que desde el año 2009 la producción académica comienza a decrecer y en 2010 y 2011 solo aparece un artículo por año (ver Figura 1).

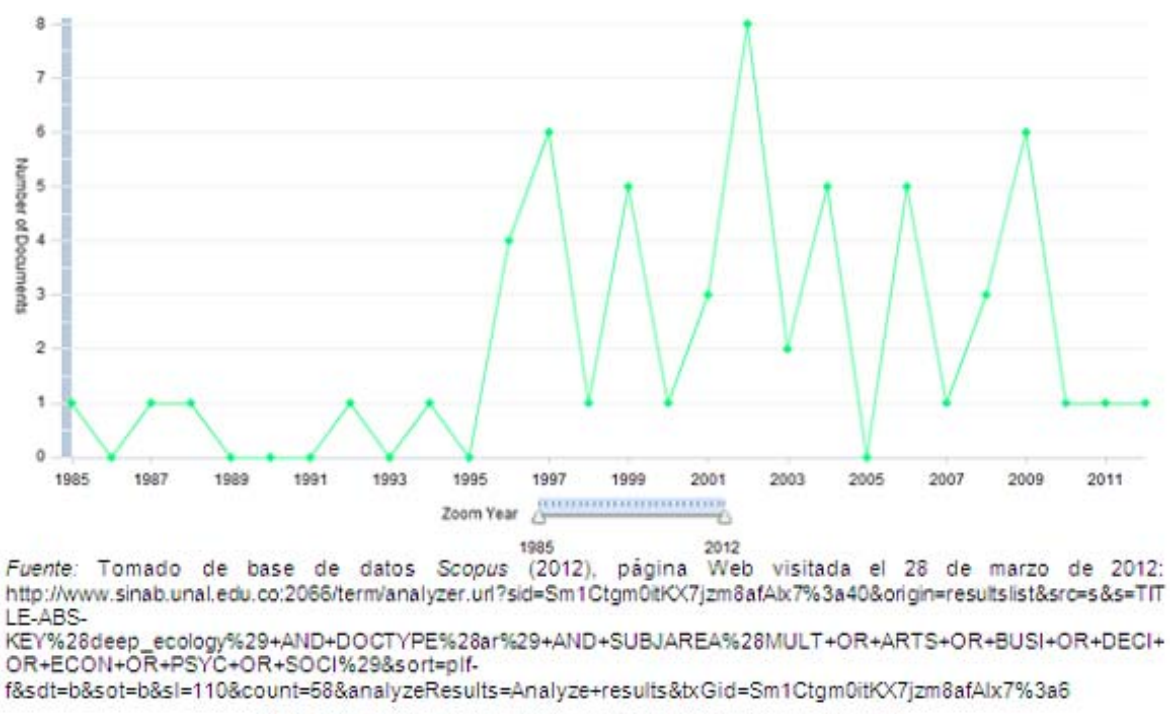

Figura 1. Publicaciones por año [Deep_ecology].

Los autores más representativos de la ecología profunda en relación con el número de artículos producidos son Markus y Taylor. El primero de estos se ocupará de mostrar los fundamentos filosóficos de la deep ecology (Markus, 2003b, 2003c, 2004), sobre todo en su artículo titulado "More-thanhuman-world. Deep ecology as environmental philosophy" (Markus, 2003c) donde presentará las diferentes opiniones de los ecologistas y aducirá que esta corriente presenta, tal vez, la crítica más contundente sobre la civilización tecnológica. El segundo de los autores, por su parte, se ocupará de relacionar la ecología profunda con la espiritualidad. Su interés fundamental es mostrar que muchos de los movimientos contraculturales -como el movimiento de la ecología profundaa pesar de rechazar la etiqueta religiosa involucran una espiritualidad de la naturaleza que permite hablar de movimientos religiosos (Taylor, 1997, 2001a, 2001b). Ahora bien, no está de más agregar que estos dos autores pese a ser los más representativos de esta corriente por haber producido 3 artículos cada uno, su producción se concentra entre el año 1997 y el año 2004. Lo que quiere decir que la deep ecology ha perdido importancia dentro de la filosofía ambiental durante los últimos años (ver Figura 2). 


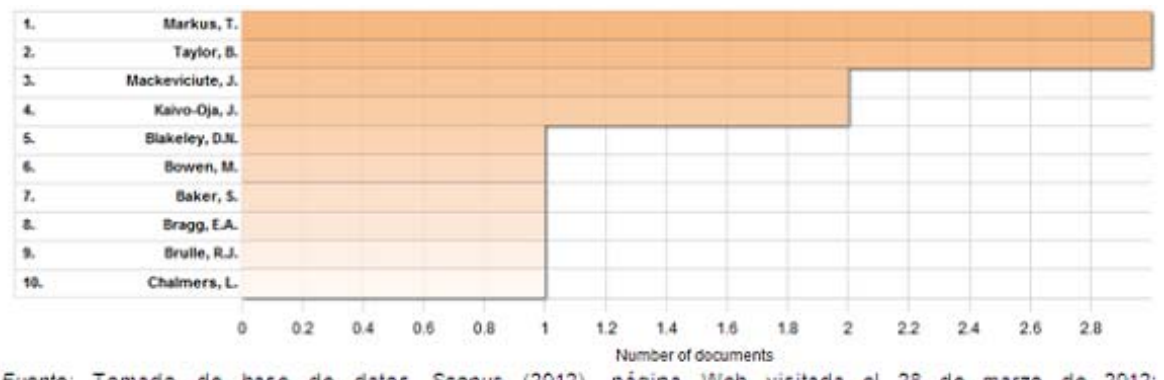

Fuente: Tomado de base de datos Scopus (2012), página Web visitacla el 28 de marzo de 2012:

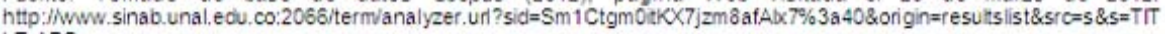
LE-ABS-

KEY\%28deep_ecology\%29+AND+DOCTYPE\%28ar\%29+AND+SUBJAREA\%28IMULT+OR+ARTS+OR+BUSI+OR+DECl+ $O R+E C O N+O \bar{R}+P S Y C+O R+S O C \mid \% 29 \& s$ ort $=p$ if -

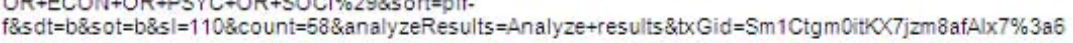

Figura 2. Publicaciones por autor [Deep_ecology]

Ahora bien, si la ecología profunda es una de las corrientes actuales de la filosofía ambiental es preciso reconocer los contextos en los que se ha concentrado la producción académica. De manera que al examinar los 58 artículos en cuestión se observa que el país en el que más se produce sobre esta corriente es en Estados Unidos, seguido del Reino Unido y Croacia (ver Figura 3). Sin duda los resultados lo evidencian, pero como se verá en las Gráficas de las otras corrientes es interesante observar que Estados Unidos es el hegemon intelectual. Hecho que lleva a pensar que, o bien las revistas Scopus presentan un sesgo hacia la literatura en inglés, o bien la mayor cantidad de estas revistas están dentro del contexto norteamericano.

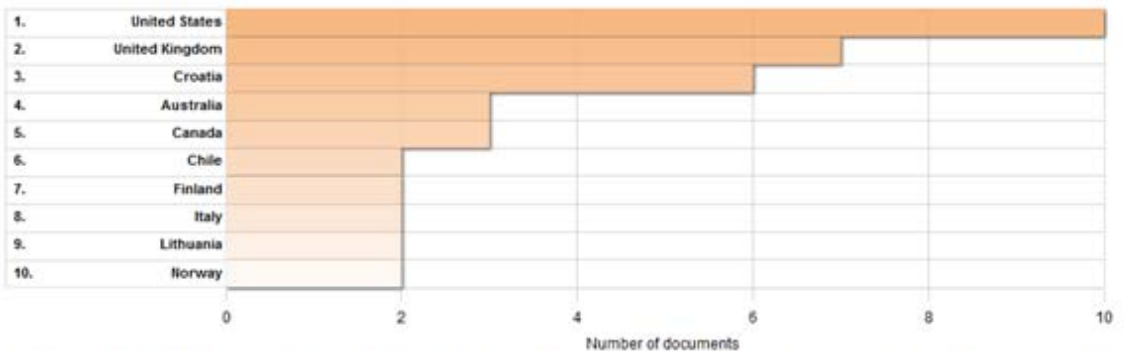

Fuente: Tomado de base de datos Scopus (2012), página Web visitada el 28 de marzo de 2012:

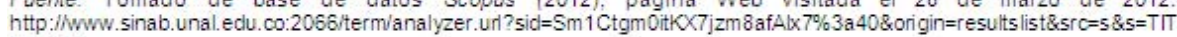
LE-ABS-

KEY\%28deep_ecology\%29+AND+DOCTYPE\%28ar\%29+AND+SUBJAREA\%28MULT+OR+ARTS+OR+BUSI+OR+DECl+ $O R+E C O N+O R+P S Y C+O R+S O C l \% 29 \&$ sort=plf-

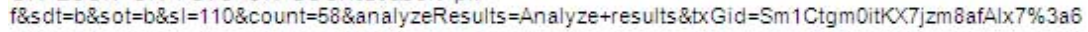

Figura 3. Publicaciones por país [Deep_ecology]

Lo cierto es que los resultados de la Figura 3 resultan relevantes en la medida que permiten inferir que a excepción de Estados Unidos no hay ningún país que esté posicionado dentro de la ecología profunda. Tal vez los resultados de Croacia y el Reino Unido sean destacables, aunque el segundo, en conformidad con el criterio del idioma confirma que el inglés es hegemónico dentro de las revistas Scopus. Mientras que el primero parece demostrar que la ecología profunda, aunque no es una corriente hegemónica $(7$ artículos no son suficientemente indicativos) tiene una importancia comparativa dentro del contexto global. 


\section{Biología de la conservación}

Al buscar dentro de las 5300 revistas de Ciencias Sociales y Humanidades bajo el criterio [Conservation_biology] se encontraron 238 artículos entre el año 1985 y el año 2012. En principio es posible afirmar que aunque el primer artículo es del año 1985, solo será hasta el año 1993 que la producción académica tendrá un cambio sustancial al aparecer publicados 6 artículos. Los años noventa representarán un crecimiento de la literatura que llegará a su pico más alto en el año 1997 con 11 artículos. Aunque, la consolidación de esta corriente solo se dará hasta la primera década del siglo XXI puesto que allí se sobrepasará la barrera de los 20 artículos anuales hasta llegar al punto más alto en el año 2011. El salto más abrupto se presenta entre el año 2002 y 2003, pasando de 10 a 20 artículos.

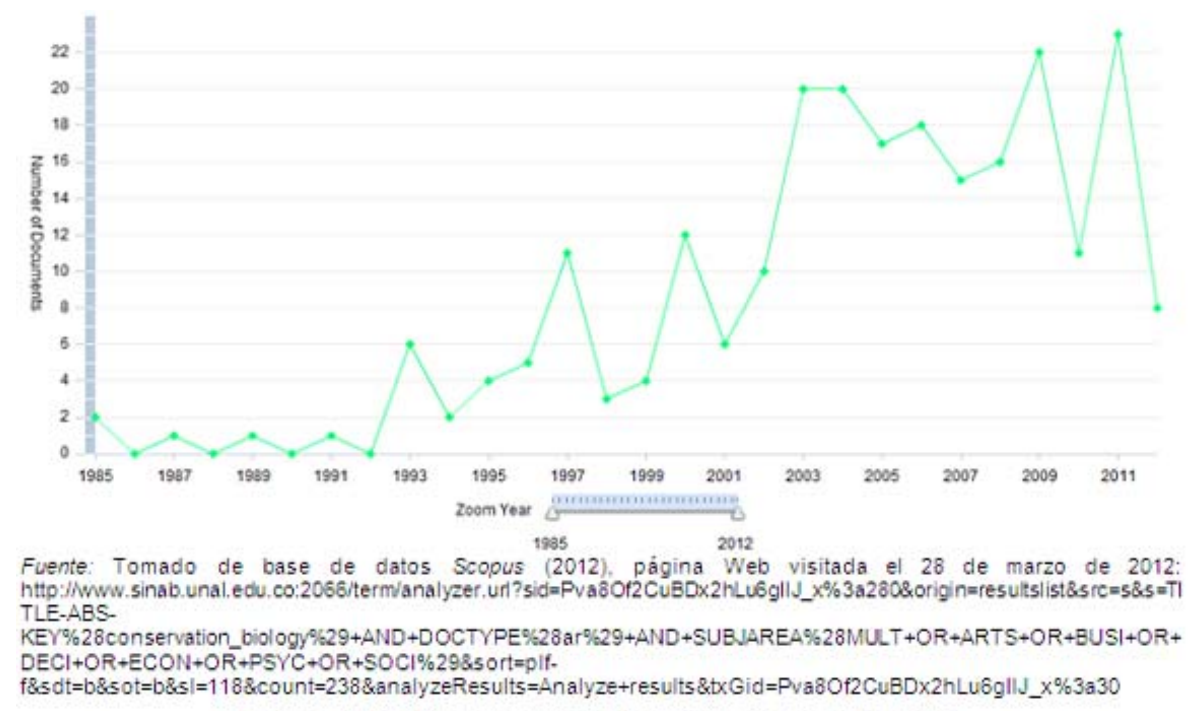

Figura 4. Publicaciones por año [Conservation_biology]

Los autores más representativos de esta corriente son Daily y Possingham con cuatro artículos publicados cada uno (ver Figura 5). El primero, vinculado a la Stanford University, puede decirse grosso modo que se ocupa de pensar el futuro de la biodiversidad y los servicios de los ecosistemas a partir de la armonización de la producción agrícola y la conservación (Sekercioglu et al., 2002; Luck et al., 2004; Armsworth et al., 2006; Chan \& Daily, 2008). Al respecto puede revisarse, por ejemplo, el trabajo titulado "The payoff of conservation investments in tropical countyside" (Chan \& Daily, 2008) en el que los autores luego de establecer que no existe un algoritmo de planificación para predecir la eficacia de las inversiones en la conservación de las tierras de cultivo, se ocupan de pensar un marco de planificación para la conservación del campo que calcula sus beneficios en la producción.

Possingham, el segundo de los autores antes mencionado, está vinculado a la University of Queensland en Australia y se ocupa, en términos generales, de pensar la conservación en el plano de la coordinación mundial y regional. A su juicio, a pesar de 
que la mayoría de los programas de conservación se aplican en el contexto nacional y subnacional, sin duda la aplicación coordinada de estos programas en contextos más amplios reduciría los costos y aumentaría el retorno de la inversión (Wilson et al., 2006; Bode et al., 2008; Chadès et al., 2008; Kark et al., 2009). En una de sus publicaciones titulada "Betweencountry collaboration and consideration of costs in crease conservation planning efficiency in the Mediterranean Basin" (Kark et al., 2009) se plantea que en el caso de los vertebrados, si cada país de la Cuenca del Mediterráneo actuará aisladamente se necesitaría un presupuesto bastante elevado para lograr los beneficios de la conservación. De manera que si existiera una acción coordinada de los países de la región se podrían ahorrar aproximadamente unos US\$67 billones, es decir, un $45 \%$ del costo total de la acción no coordinada. En estos términos, es posible inferir en relación con los trabajos de Daily y Possingham que la preocupación de la biología de la conservación se concentra, al menos en los trabajos explorados, en una funcionalización de la preservación del medio a partir de máximas mercantiles. Hecho que lleva a pensar en una corriente filosófica acentuada sobre presupuestos antropocéntricos, desde los que se piensa que aunque el medio ambiente no puede dejar de explotarse, sí es posible acompañar la explotación de una rigurosa conservación.

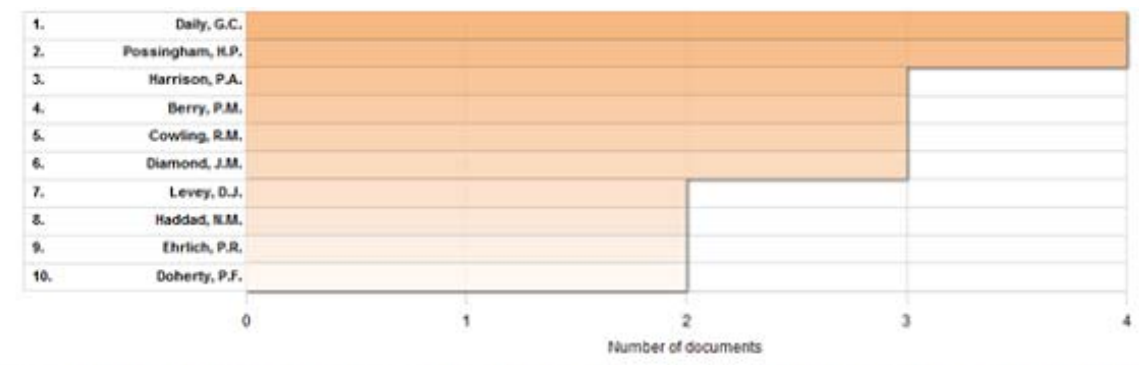

Fuente: Tomado de base de datos Scopus (2012), página Web visitada el 28 de marzo de 2012:

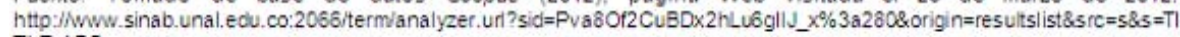

KLE-ABS. $D E C l+O R+E C O N+O R+P S Y C+O R+S O C l \% 298$ s ort $=p$ lf-

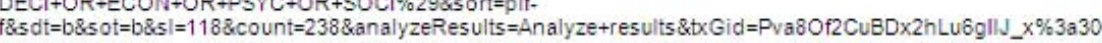

Figura 5. Publicaciones por autor [Conservation_biology].

Reconociendo la importancia de la biología de la conservación dentro del campo de la filosofía ambiental, es preciso atender ahora a los contextos en los que ha proliferado esta corriente. De este modo, el país en el que se ha dado una mayor producción académica dentro de este campo es Estados Unidos, hecho que lleva a pensar en los mismos cuestionamientos que se hicieron al hablar de la ecología profunda pues la diferencia entre este país y el Reino Unido, que es el segundo país en la Figura 6, es de aproximadamente 80 artículos. En este sentido se puede deducir que existe una concentración de la literatura acompañada por una producción exacerbada de artículos sobre esta corriente dentro de los países de habla inglesa, a saber, Estados Unidos (primero en la lista), Reino Unido (segundo en la lista), Australia (tercero en la lista) y Canadá (cuarto en la lista). Aunque esta característica podría ser explicada en función del número de revistas Scopus en inglés, lo que resulta particularmente interesante es el 
crecimiento exponencial de la literatura sobre todo en el caso de Estados Unidos.

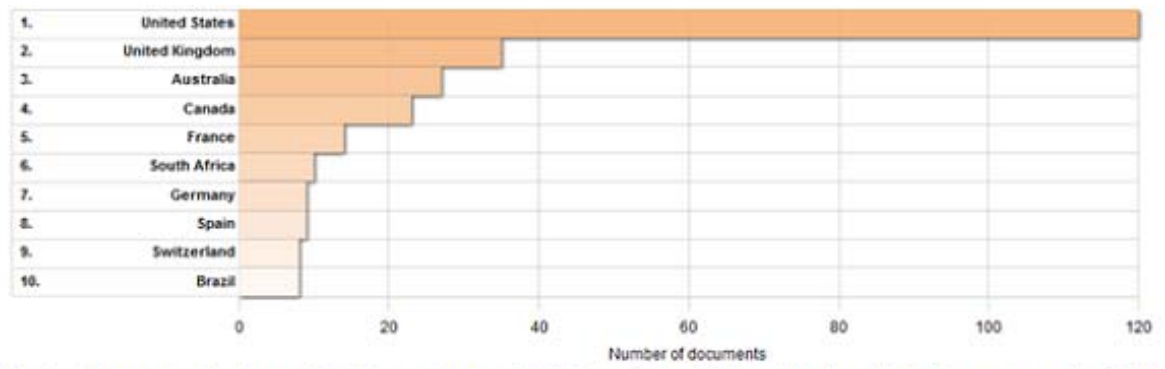

Fuente: Tomado de base de datos Scopus (2012), página Web visitada el 28 de marzo de 2012

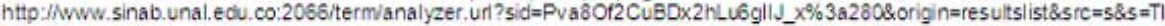
TLE-ABS-

KEV\%28conservation_biology\%29+AND+DOCTVPE\%28ar\%29+AND+SUBUAREA\%28MULT+OR+ARTS+OR+BUSI+OR+ $D E C l+O R+E C O N+O R+P S Y C+O R+S O C 1 \% 298$ s ort $=$ if.

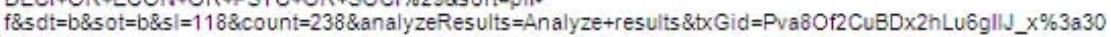

Figura 6. Publicaciones por pais [Conservation_biology].

Dicho lo anterior, no solo es de destacar la hegemonía intelectual de los países de habla inglesa en esta corriente, sino además su enorme relevancia durante los últimos años. Tal parece que la literatura sobre filosofía ambiental le ha dado un rol preponderante a esta corriente por encima de otras. Quizás habría que examinar el marcado desbalance en la producción entre los países europeos y norteamericanos, con respecto a los países latinoamericanos y africanos. Es de destacar que solo aparecen en la lista Suráfrica y Brasil.

\section{Biorregionalismo}

Continuando con los parámetros de búsqueda de las corrientes filosóficas anteriores, en la base de datos Scopus se hallaron 27 artículos entre 1985 y 2012. En principio es preciso señalar que la producción académica de esta corriente es notablemente inferior a la de las corrientes anteriores, y aunque su primer artículo aparece registrado en 1989 va a haber un periodo de sequía intelectual hasta 1997, es decir, casi una década. Hecho que lleva a pensar que en realidad esta corriente es bastante reciente y va a tener su auge hacia finales de los noventa y la primera década de 2000. Igualmente es de destacar que no existen picos muy altos, sino que por el contrario la producción es constante, de tal forma que entre 1997 y 2012 todos los años aparecen al menos con 1 artículo publicado, y el año 2007 aparece con el pico de mayor producción con 4 artículos. Es así que, teniendo en cuenta los artículos publicados en revistas Scopus es posible afirmar que el biorregionalismo, en tanto corriente filosófica, tiene una existencia reciente y de poca relevancia. 


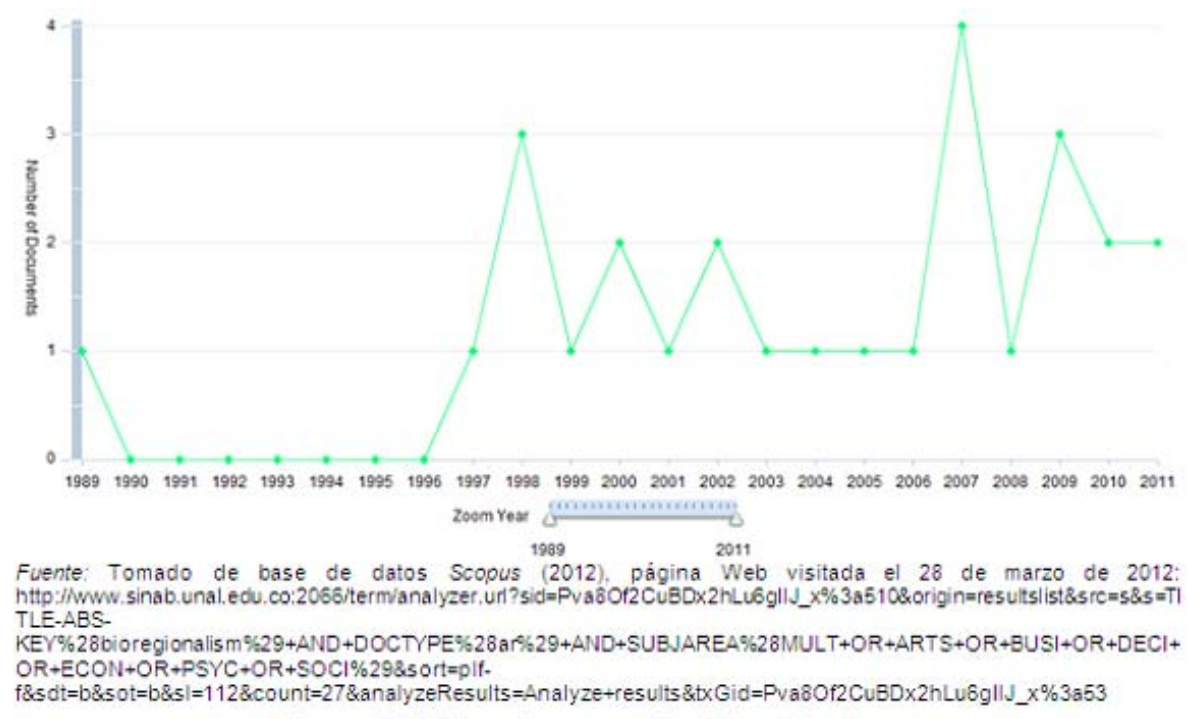

Figura 7. Publicaciones por año [Bioregionalism].

Lo anterior es posible demostrarlo también, haciendo referencia al número de artículos publicados por autor. La Figura 8 muestra que los 27 artículos publicados sobre biorregionalismo han sido escritos por autores diferentes, es decir que no hay ningún autor representativo de esta corriente. Sin embargo, pese a que no hay ningún autor representativo es de destacar el trabajo de Rowan Gray y de Richard Evanoff. El primero de estos, debido a que en su artículo titulado "Practical bioregionalism: A philosophy for a sustainable future and hypothetical transition strategy for Armidale, New South Wales, Australia" (Gray, 2007) presenta desde un "enfoque ecoutópico" un conjunto hipotético de estrategias de transición encaminadas a transformar una comunidad regional de Australia. Lo interesante es que allí el autor logra combinar principios biorregionales con la teoría de la modernización ecológica; y el segundo, a su vez, porque en su artículo "Bioregionalism and cross-cultural dialogue on a land ethic" (Evanoff, 2007) presenta una postura a favor de la armonización del biorreginalismo y la interculturalidad, es decir que el autor logra confrontar la visión de una ética del medio ambiente mundial, sobre la base de una ética ajustada a la perspectiva biorregional con connotaciones globales a partir de diálogos interculturales con diferentes entornos locales. 


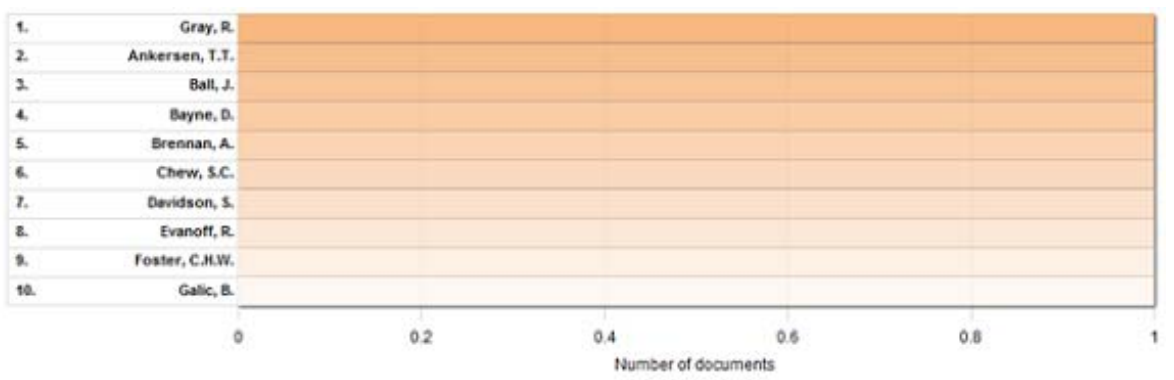

Fuente: Tomado de base de datos Scopus (2012), página Web visitada el 28 de marzo de 2012:

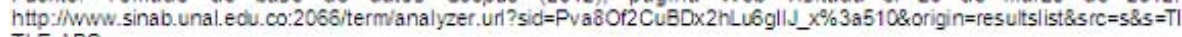
TLE-ABS-

KEV\%28bioregionalism\%29+AND+DOCTYPE\%28ar\%29+AND+SUBJAREA\%28MULT+OR+ARTS+OR+BUS1+OR+DECI+ $O R+E C O N+O R+P S Y C+O R+S O C l \% 298$ s ort $=$ if-

$f \& s d t=b \& 50 t=b \& s \mid=112 \&$ count $=27$ \&analyzeResults=Analyze+results\&txGid=Pva8Of2CuBD 2 hLu6gllJ_x\%3a53

Figura 8. Publicaciones por autor [Bioregionalism].

De este modo, dado que esta corriente no tiene una producción prolífica, resulta presumible pensar que no hay un hegemon intelectual. Sin embargo, al examinar la Figura 9 se observa que 8 de los 27 artículos fueron producidos por Estados Unidos, mientras que el segundo país de la lista, Australia, solo aparece con 3. En este sentido, a pesar de que el biorregionalismo tiene una modesta producción de artículos, existe una concentración de la literatura en Estados Unidos, en particular, y en países de habla inglesa, en general.

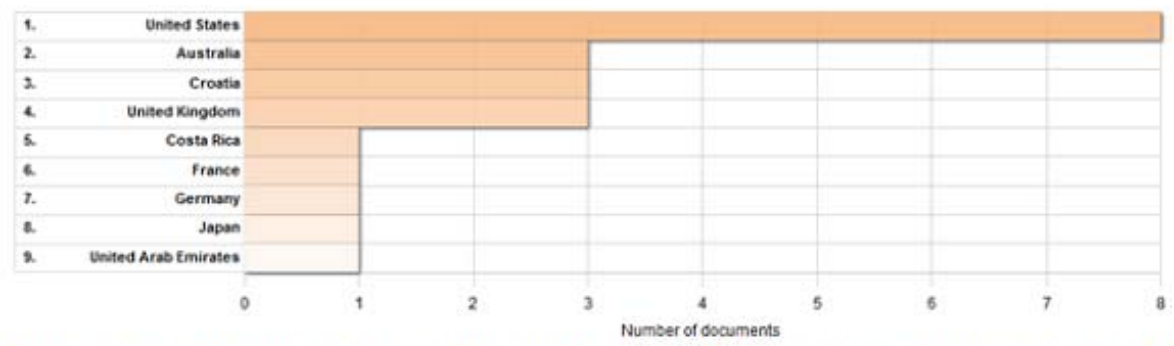

Fuente: Tomado de base de datos Scopus (2012), página Web visitada el 28 de marzo de 2012 :

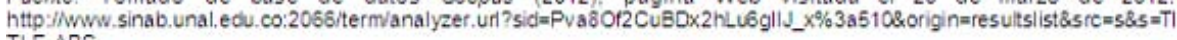
TLE-ABS-

KEV\%28bioregionalism\%29+AND+DOCTYPE\%28ar\%29+AND+SUBJAREA\%28MULT+OR+ARTS+OR+BUSI+OR+DECl+ $O R+E C O N+O R+P S Y C+O R+S O C 1 \% 29 \&$ s ort $=p$ if-

$f \& s d t=b \& s$ ot $=b \& s \mid=112 \&$ count=27 \&analyzeResults=Analyze+results\&tx Gid=Pva8Of2CuBD 2 hLu6gllJ_x\%3a53

Figura 9. Publicaciones por pais [Bioregionalism].

Ciertamente una constante de esta gráfica con respecto a las anteriores es que vuelve a evidenciarse la hegemonía intelectual de Estados Unidos. Es así que resulta pertinente volver sobre un cuestionamiento ya planteado: ¿Las revistas Scopus muestran un sesgo hacia los países de habla inglesa? Sin duda, este cuestionamiento escapa a las pretensiones de este artículo, aunque sin duda los resultados explorados evidencian una desproporcional producción de estos países con respecto a los demás. De manera que aun a pesar del sesgo, es muy residual la producción de los países que no son de habla inglesa. Razón por la cual, es factible pensar que el desarrollo de esta corriente no solo ha sido subsidiario, sino que además ha estado concentrado en unos contextos determinados, en los que sin duda no ha sido central desde el punto de vista de la filosofía ambiental. 


\section{Ecofeminismo}

Manteniendo los criterios de búsqueda de las corrientes filosóficas anteriores se encuentran 118 artículos entre 1985 y 2012. Estos resultados permiten afirmar que el ecofeminismo, no solo es una corriente reciente en la medida que el primer artículo que aparece registrado es del año 1993; sino que además es una corriente en ascenso debido a que en los últimos años muestra una producción constante y prolífica (ver Figura 10). Es de anotar que esta corriente va a tener un auge hacia finales de los años noventa y luego a finales de la primera década de 2000, y aunque las publicaciones mantienen un crecimiento desde el año 1993, se va a dar un rezago entre el 2002 y el 2005, para luego tener el pico más alto en 2010 con 13 artículos.

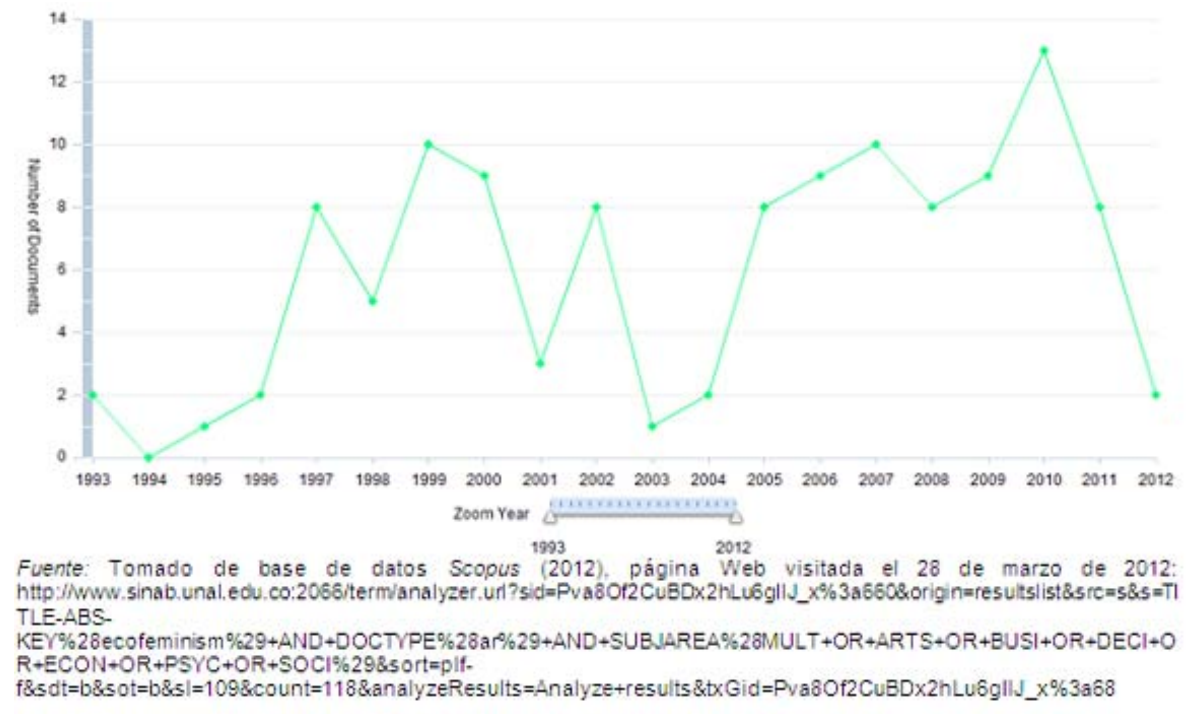

Figura 10. Publicaciones por año [Ecofeminism].

En relación con los autores más representativos de esta corriente, es preciso destacar, por un lado, a Marija Geiger que se ha ocupado de mostrar la estrecha relación entre lo local y lo global, analizando el potencial del lugar en la promoción de la sostenibilidad en el contexto de un mundo en constante globalización (Geiger \& Zeman, 2010), dejando al mismo tiempo de presente la relación entre el medio ambiente y la religión, a partir de la articulación entre la sociedad patriarcal, el cristianismo y el antropocentrismo, en orden a proponer una visión matriarcal que desde el ecofeminismo reivindique el papel de la madre tierra (Geiger, 2002; Galić \& Geiger, 2007); y por otro lado, a Ariel Salleh que ha trabajado el asunto del feminismo desde la perspectiva sociológica dejando de presente que muchas de las corrientes contemporáneas han surgido como resultado de la relación del ser humano con la naturaleza. Sin embargo, esta relación se ha visto envuelta dentro del fenómeno marxista de la alienación, pues con la entrada de la industrialización las personas pierden el sentido de su propia naturaleza orgánica. Lo cual en otras palabras significa que el hombre se encuentra escindido de su propia 
naturaleza y no se reconoce a sí mismo (Salleh, 1996, 2003, 2009).

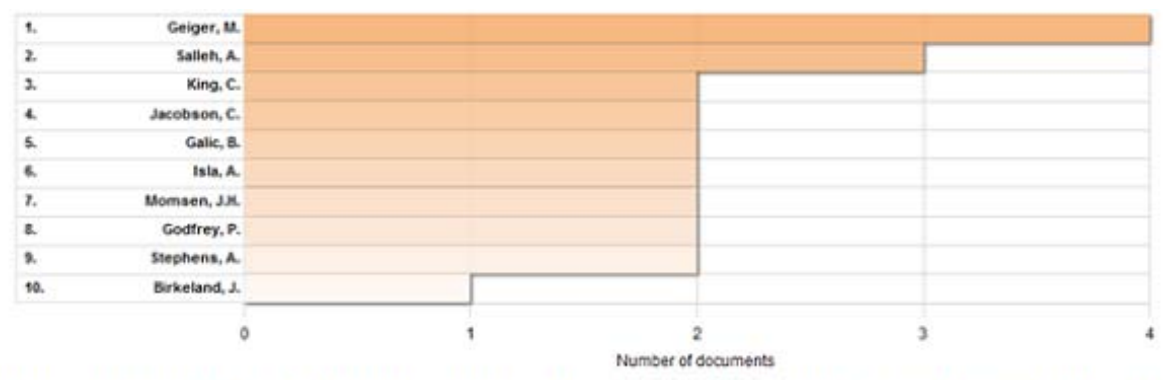

Fuente: Tomado de base de datos Scopus (2012), página Web visitada el 28 de marzo de 2012:

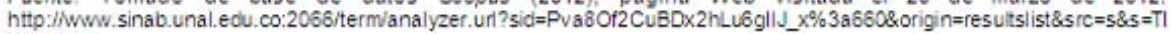
TLE-ABS-

KEV\%28ecofeminism\%29+AND+DOCTVPE\%28ar\%29+AND+SUBUAREA\%28MULT+OR+ARTS+OR+BUSI+OR+DECI+O $R+E C O N+O R+P S Y C+O R+S O C l \% 298 s$ ort $=$ plif-

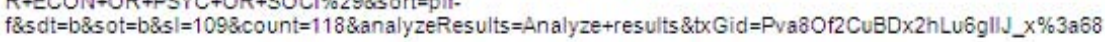

Figura 11. Publicaciones por autor [Ecofeminism]

Ahora bien, reconociendo que el ecofeminismo a diferencia de las otras corrientes rompe con el status quo, al propugnar por una mirada del medio ambiente desde la perspectiva del sujeto excluido, resulta preciso preguntarse si esta visión alternativa ha tenido un auge en los países del primer mundo, y sobre todo, en Estados Unidos. En ese sentido, al examinar la Figura 12 se observa que, al igual que en las Gráficas de las anteriores corrientes, la hegemonía de Estados Unidos frente a los demás países es notable. En este caso se observa que 30 de los 118 artículos pertenecen a este país, mientras que el Reino Unido, Australia y Canadá se sitúan en el segundo, tercer y cuarto lugar, respectivamente. En este sentido, se vuelve a evidenciar una hegemonía de los países de habla inglesa, lo que reafirma los cuestionamientos planteados con anterioridad. Sin embargo, es de destacar que países como Brasil, Argentina, China e India, también entran en el listado, lo cual implica que la hegemonía no es completa e incluye países históricamente excluidos de los circuitos de conocimiento.

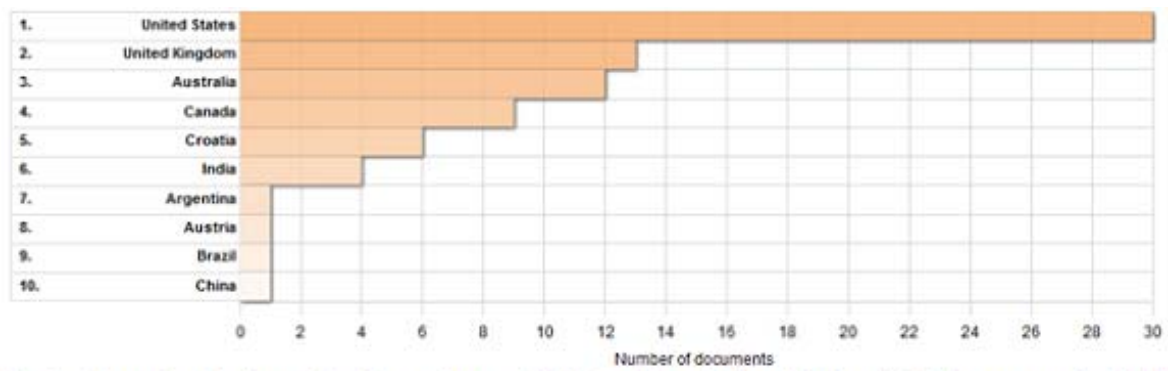

Fuente: Tomado de base de datos Scopus (2012), página Web visitada el 28 de marzo de 2012

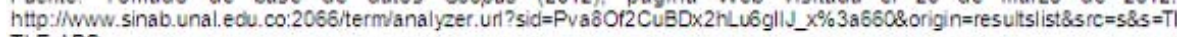
TLE-ABS-

KEV\% 28 ecofeminism\%29+AND+DOCTVPE\%28ar\%29+AND+SUBUAREA\%28MULT+OR+ARTS+OR+BUSI+OR+DECI+O $R+E C O N+O R+P S Y C+O R+S O C l \% 29 \& 5$ ort $=$ plif-

f\&sdt=b\&sot=b\&s $=109 \&$ count=118\&analyzeResults=Analyze+results\&txGid=Pva80f2CuBD 2 hLu6gllJ_x\%3a68

Figura 12. Publicaciones por pais [Ecofeminism].

Quizás lo que resulta más sorprendente del ecofeminismo es que representa la visión más novedosa y recalcitrante de las cuatro corrientes aquí examinadas, sobre todo en razón de que traduce el problema de la mujer en tanto sujeto excluido, 
evidenciado en el rol emancipatorio del feminismo, para situarlo en el plano del medio ambiente, en orden a irrumpir con las lógicas antropocéntricas para instaurar una mirada ecocéntrica. En este sentido, es preciso reconocer que el ecofeminismo, pese a ser una corriente reciente, ha cobrado importancia en los últimos años de tal manera que se encuentra por encima de corrientes como el biorregionalismo y la ecología profunda.

\section{CONCLUSIÓN}

A lo largo de este artículo se buscó indagar por un campo residualmente explorado dentro de los estudios ambientales: la filosofía ambiental, que pese a las críticas por su falta de utilidad, es dejado a un lado y en muchos casos no es tomado en consideración. Sin embargo, contrario a esta visión pesimista, este artículo tomó como punto de partida que la filosofía ambiental es el insumo básico para cualquier concepción sobre el ambiente, de manera que un rezago en esta materia bien puede significar una posición frívola o superficial. Sobre esta base, se evidenció un gran vacío en los trabajos publicados sobre filosofía ambiental, y más aun, en los trabajos sobre filosofía de la educación ambiental. Razón por la cual, la posición de quien escribe, más allá de exhortar a los estudiosos del medio ambiente a trabajar en el campo de la filosofía, fue plantear a manera de hipótesis que el vacío de la literatura se debía a que la gran mayoría de trabajos han girado en torno a las corrientes específicas de la filosofía ambiental.

Por esta razón, el primer apartado se ocupó de examinar los residuales trabajos sobre filosofía de la educación ambiental, en orden a identificar las corrientes que sobre la materia han expuesto diferentes autores. De allí resulta pertinente rescatar trabajos como el de Walter (2009) o el de Corcoran y Sievers (1994) en la medida que permiten dilucidar un campo sobre el que diferentes académicos en la materia han tomado partido. En otras palabras, estos trabajos permiten ver de manera sintética las diferentes concepciones sobre el ambiente. Por ende, este apartado deja como resultado dos reflexiones: por un lado, una preocupación por la escasa reflexión teórica acerca de la filosofía ambiental como campo dentro de los estudios ambientales; y por otro lado, una síntesis de las principales corrientes de la filosofía de la educación ambiental.

En cuanto al segundo apartado, se puede decir que este artículo buscó presentar un estado del arte sobre la filosofía ambiental, en orden a clasificar la literatura en unas categorías que dieran cuenta de las tendencias generales. Es de anotar que un primer hallazgo es que aun cuando hay más trabajos de filosofía ambiental que de filosofía de la educación, ciertamente las reflexiones siguen siendo aisladas. En cuanto a la clasificación que se hizo fue posible observar que una de las principales preocupaciones de aquellos que han trabajado sobre el tema es el asunto de los enfoques. Razón por la cual sobre la base de este hallazgo se justifica la hipótesis, y en 
consecuencia el tercer apartado, a saber, que la búsqueda de artículos por enfoque aumenta exponencialmente el número de trabajos.

Es así que, finalmente, en el último apartado se logra demostrar que efectivamente la búsqueda de artículos por corrientes específicas de la filosofía ambiental aumenta los resultados. Lo cual deja de presente que la filosofía ambiental construye los cimientos de una concepción sobre el ambiente, aunque paradójicamente las reflexiones epistemológicas sobre las corrientes en general son escasas. Lo que quiere decir que los autores asumen posturas y las justifican con estas corrientes pero no presentan trabajos en los que se debatan otras concepciones divergentes.

De manera que el resultado de este estudio bibliométrico con el que culmina el artículo, es que las corrientes de mayor importancia, en términos del número de artículos publicados, son la biología de la conservación y el ecofeminsimo. Dos posturas que resultan antagónicas en la medida que una se asocia a una visión conservacionista e incluso neoliberal y otra se asocia a una visión alternativa e incluso insurgente. Sin embargo, lo que resulta interesante del estudio es que deja de presente la discusión acerca de las posturas que hoy día se tienen acerca del ambiente: unas que se armonizan con el mercado y otras que lo rechazan a cabalidad. Es la posición, del sujeto del primer mundo y la posición del sujeto excluido. Aunque lo que resulta aun más paradójico es que la mayor producción en todas las corrientes provino de los Estados Unidos, lo que lleva a indagar con mayor detenimiento el sesgo hacia los países de habla inglesa de Scopus.

En suma, por todo lo anterior, se puede afirmar que en el campo de los estudios ambientales resulta preponderante el papel de la filosofía ambiental. De manera que un trabajo como el que aquí se presenta debe ser propedéutico y debe servir como invitación para que las concepciones sobre el ambiente estén afincadas en reflexiones serias y posturas críticas. De manera que el papel del medio ambiente, puede ser interpretado como el rol de un sujeto excluido en función del humanismo degradante que desde visiones antropocéntricas han situado el valor de la naturaleza en función de la explotación de sus recursos. Por esa razón se justifica el cada vez más importante papel de posturas como el ecofeminismo y se impele a los defensores de posturas como la biología de la conservación a que afiancen sus argumentos y contrasten sus posturas con estas nuevas corrientes que están emergiendo. 


\section{BIBLIOGRAFÍA}

- Anthony, R. (2010). Building a Sustainable Future for Animal Agriculture: An Environmental Virtue Ethic of Care Approach within the Philosophy of Technology. Journal of Agricultural and Environmental Ethics, 25(2), 123-144.

- Armsworth, P. R.; Daily, G. C.; Kareiva, P. y Sanchirico, J. N. (2006). Land market feedbacks can undermine biodiversity conservation. Proceedings of the National Academy of Sciences of the United States of America, 103(14), 5403-5408.

- Bode, M.; Wilson, K. A.; Brooks, T. M.; Turner, W. R.; Mittermeier, R. A.; McBride, M. F.; Underwood, E. C. et al. (2008). Cost-effective global conservation spending is robust to taxonomic group. Proceedings of the National Academy of Sciences of the United States of America, 105(17), 6498-6501.

- Chadès, I.; McDonald-Madden, E.; McCarthy, M. A.; Wintle, B.; Linkie, M. y Possingham, H. P. (2008). When to stop managing or surveying cryptic threatened species. Proceedings of the National Academy of Sciences of the United States of America, 105(37), 13936-13940.

- Chan, K. M. A. y Daily, G. C. (2008). The payoff of conservation investments in tropical countryside. Proceedings of the National Academy of Sciences of the United States of America, 105(49), 19342-19347.

- Corcoran, P. y Sievers, E. (1994). Reconceptualizing environmental education: five possibilities. Journal of Environmental Education, 25, 4-8. doi:10.1080/00958964.1994.9941958

- De Laplante, K. (2004). Can you teach environmental philosophy without being an environmentalist? Worldviews: Environment, Culture, Religion, 8(2-3), 198-212.

- Elias, J. y Merriam, S. (1995). Philosophical foundations of adult education (2nd ed.). Malabar FL: Kreiger.

- Evanoff, R. (2007). Bioregionalism and cross-cultural dialogue on a land ethic. Ethics, Place and Environment, 10(2), 141-156.

- Galić, B. y Geiger, M. (2007). From domination logic toward ethics of care - Conceptual foundations of ecofeminism. Socijalna Ekologija, 16(1), 17-33.

- Geiger, M. (2002). Spiritual aspects of ecofeminism. Socijalna Ekologija, 11(1-2), 15-27.

- Geiger, M. y Zeman, Z. (2010). Place of living and integral sustainability - Vitality of local in a globalized world. Drustvena Istrazivanja, 19(3), 377-400.

- Gold, J. R.; Revill, G. y Haigh, M. J. (1996). Interpreting the dust bowl: Teaching environmental philosophy through film. Journal of Geography in Higher Education, 20(2), 209-221.

- Gómez, M. y De Puig, I. (2003). Ecodiálogo, Environmental Education and Philosophical Dialogue. The Journal of Philosophy for Children, 16(4), 37-40. 
- Goralnik, L. y Nelson, M. P. (2011). Framing a philosophy of environmental action: Aldo Leopold, John Muir, and the importance of community. Journal of Environmental Education, 42(3), 181-192.

- Gray, R. (2007). Practical bioregionalism: A philosophy for a sustainable future and a hypothetical transition strategy for Armidale, New South Wales, Australia. Futures, 39(7), 790-806.

- Hotam, Y. (2010). Ecology and Pedagogy: On the educational implications of postwar environmental philosophy. Policy Futures in Education, 8(3-4), 478487.

- Howarth, R. B. (2007). Adaptive management and the philosophy of environmental policy. Perspectives in Biology and Medicine, 50(3), 453-458.

- Inhaber, H. (1975). Philosophy and limitations of environmental indices. Social Indicators Research, 2(1), 39-51.

- James, G. A. (1998). The construction of India in some recent environmental philosophy. Worldviews: Environment, Culture, Religion, 2(1), 3-20.

- Kark, S.; Levin, N.; Grantham, H. S. y Possingham, H. P. (2009). Between-country collaboration and consideration of costs increase conservation planning efficiency in the Mediterranean Basin. Proceedings of the National Academy of Sciences of the United States of America, 106(36), 15368-15373.

- Kennet, M. (2007). Editorial: Progress in Green Economics: Ontology, concepts and philosophy. Civilisation and the lost factor of reality in social and environmental justice. International Journal of Green Economics, 1(3-4), 225-249.

- Li, P.; Huang, Z.; Ren, H.; Liu, H. y Wang, Q. (2010). The Evolution of Environmental Management Philosophy Under Rapid Economic Development in China.

- . (2011). The evolution of environmental management philosophy under rapid economic development in China. Ambio, 40(1), 88-92.

- Light, A. (2002). Contemporary environmental ethics from Metaethics to public philosophy. Metaphilosophy, 33(4), 427-449.

- Lübbe-Wolff, G. (2001). Efficient environmental legislation - On different philosophies of pollution control in Europe. Journal of Environmental Law, 13(1), 75-86.

- Luck, G. W.; Rickettst, T. H.; Daily, G. C. y Imhoff, M. (2004). Alleviating spatial conflict between people and biodiversity. Proceedings of the National Academy of Sciences of the United States of America, 101(1), 182186.

- Macauley, D. (2005). The flowering of environmental roots and the four elements in Presocratic philosophy: From Empedocles to Deleuze and Guattari. Worldviews: Environment, Culture, Religion, 9(3), 281-314.

- Markus, T. (2003a). More-than-human-world. Deep ecology as environmental philosophy. Socijalna Ekologija, 12(3-4), 143-163. 
-

(2003b). "A head into nowhere". Lewis Mumford and the ecological criticism of technological civilization. Socijalna Ekologija, 12(1-2), 27-45.

- $\quad$ (2003c). More-than-human-world. Deep ecology as environmental philosophy. Socijalna Ekologija, 12(3-4), 143-163.

- _ (2003d). More-than-human-world. Deep ecology as environmental philosophy. Socijalna Ekologija, 12(3-4), 143-163.

- . (2004). Ecology and biology: Environmental implications of contemporary biological theories. Socijalna Ekologija, 13(2), 129-151.

- Nash, C. (1999). Environmental history, philosophy and difference: Feminist perspectives on historical and contemporary human-environmental relations. Documents d'Analisi Geografica, (35), 61-72.

- . (2000). Environmental history, philosophy and difference. Journal of Historical Geography, 26(1), 2327.

- Park, C. D. (1984). Towards a philosophy of environmental education. Environmental Education \& Information, 3(1), 3-15.

- Parkin, J. (1992). A philosophy for multiattribute evaluation in environmental impact assessments. Geoforum, 23(4), 467-475.

- Pfister, L. F. (2007). Environmental ethics and some probing questions for traditional Chinese philosophy. Journal of Chinese Philosophy, 34(Suppl.), 101-123.

- Plumwood, V. (1999). The struggle for environmental philosophy in Australia. Worldviews: Environment, Culture, Religion, 3(2), 157-178.

- Salleh, A. (1996). Social ecology and "the man question". Environmental Politics, 5(2), 258-273.

- (2003). Ecofeminism as sociology. Capitalism, Nature, Socialism, 14(1), 61-74.

- . (2009). The dystopia of technoscience: An ecofeminist critique of postmodern reason. Futures, 41(4), 201-209.

- Schultz, J. R.; Simpson, S. y Elfessi, A. M. (2011). The environmental action and philosophy matrix: An exploratory study of the environmental attitudes of recreation management and environmental studies students. Journal of Environmental Education, 42(2), 98-108.

- Scott, D. (2004). Transforming the "Market-Model University": Environmental philosophy, citizenship and the recovery of the humanities. Worldviews: Environment, Culture, Religion, 8(2-3), 162-184.

- Sekercioglu, Ç. H.; Ehrlich, P. R.; Daily, G. C.; Aygen, D.; Goehring, D. y Sandí, R. F. (2002). Disappearance of insectivorous birds from tropical forest fragments. Proceedings of the National Academy of Sciences of the United States of America, 99(1), 263-267.

- Taylor, B. (1997). Earthen spirituality or cultural genocide? Radical environmentalism's appropriation of Native American spirituality. Religion, 27(2), 183-215. 
(2001a). Earth and nature-based spirituality (Part I): From deep ecology to radical environmentalism. Religion, 31(2), 175-193.

- . (2001b). Earth and nature-based spirituality (Part II): From earth first! and bioregionalism to scientific paganism and the new age. Religion, 31(3), 225-245.

- Thompson, P. B. y Whyte, K. P. (2011). What Happens to Environmental Philosophy in a Wicked World? Journal of Agricultural and Environmental Ethics, Forthcoming.

- Tyburski, W. (2008). Origin and development of ecological philosophy and environmental ethics and their impact on the idea of sustainable development. Sustainable Development, 16(2), 100-108.

- Utsler, D. (2009). Paul Ricoeur's hermeneutics as a model for environmental philosophy. Philosophy Today, 53(2), 173-178.

- Walter, P. (2009). Philosophies of adult environmental education. Adult Education Quarterly, 60(1), 3-25.

- Wilson, K. A.; McBride, M. F.; Bode, M. y Possingham, H. P. (2006). Prioritizing global conservation efforts. Nature, 440(7082), 337-340.

1. Profesor de Tiempo Completo, Facultad de Educación, Universidad Antonio Nariño. Miembro del Grupo Conciencia de la misma Universidad, y del grupo de Investigación Cultura Jurídico-Política, Instituciones y Globalización de la Universidad Nacional de Colombia. Magíster en Estudios Políticos del Instituto de Estudios Políticos y Relaciones Internacionales de la Universidad Nacional de Colombia.aquiloc@yahoo.com.ar 